\title{
SYSTEMS OF ORDINARY DIFFERENTIAL EQUATIONS WHICH GENERATE AN ORDER PRESERVING FLOW.
} A SURVEY OF RESULTS *

\author{
HAL L. SMITH $\dagger$
}

\begin{abstract}
This article consists of a survey of results concerning the qualitative behavior of solutions of systems of ordinary differential equations which generate an order preserving flow. We restrict our consideration to partial orderings on $R^{n}$ induced by any one of its orthants; a flow preserves ordering if any two solutions $x(t)$ and $y(t)$ are ordered, $x(t) \leqq y(t)$, for all $t>0$ whenever $x(0) \leqq y(0)$. Many of the important results for such systems have only recently been obtained, principally by $M$. W. Hirsch, who pointed out the tendency of their solutions to converge to equilibrium. Less well known are some global geometric constraints on the stable manifold of an equilibrium and the existence of heteroclinic orbits connecting ordered equilibria. A particularly striking result for this class of systems is the easily computable necessary and sufficient condition for stability of an equilibrium.

One of our main goals is to show that by allowing partial orderings on $R^{n}$ generated by orthants other than the positive one, the usual restrictive Kamke (quasimonotone) condition (all "off-diagonal" feedbacks are positive) which results from the standard ordering is modified in such a way as to allow (selectively) some negative feedback. As a consequence, there are many interesting and nontrivial applications of the theory.

Although the focus of this paper is on systems of ordinary differential equations, some recent comparison results, derived by Conway and Smoller, for systems of reaction-diffusion equations fit quite naturally in our framework and are reviewed.
\end{abstract}

Key words. cooperative system, Kamke (quasimonotone) condition, order preserving flow

AMS(MOS) subject classifications. $34 \mathrm{~A} 40,34 \mathrm{C} 11,34 \mathrm{C} 35$

1. Introduction. The purpose of this paper is to give a survey of some results for systems of ordinary differential equations in $R^{n}$ which have the property that the flow preserves a partial ordering " $\leqq_{K}$ " in $R^{n}$ generated by one of the orthants, $K$ of $R^{n}$. More precisely, we consider here autonomous systems of ordinary differential equations:

$$
x^{\prime}=f(x), \quad x \in U \subseteq R^{n}
$$

where $f$ is a continuously differentiable function defined in a suitable open set $U$ of $R^{n}$. We write $\phi_{t}(x)$ for the solution $x(t)$ of (1.1) which satisfies $x(0)=x$. Let $K$ be an orthant of $R^{n}, K=\left\{x \in R^{n}:(-1)^{m_{i}} x_{i} \geqq 0,1 \leqq i \leqq n\right\}$, where $m_{i} \in\{0,1\}$. Then $K$ is a cone in $R^{n}$ and, as such, generates a partial ordering " $\leqq_{K}$ " in $R^{n}$ in the usual fashion: $x \leqq_{K} y$ if and only if $y-x \in K$. We say that the flow of (1.1) preserves $\leqq_{K}$ in case that whenever $x$ and $y$ lie in $U$ and $x \leqq_{K} y$ then $\phi_{t}(x) \leqq_{K} \phi_{t}(y)$ for all $t \geqq 0$ for which both solutions are defined.

The well-known result, often attributed to Kamke [27] but in fact due to Müller [35], gives sufficient conditions for the flow of (1.1) to preserve the usual partial ordering on $R^{n}$ induced by the cone $R_{+}^{n}$. These conditions are roughly that $f_{i}$ is nondecreasing in $x_{j}$ for $j \neq i$ in $U$. This well-known result was extended to give sufficient conditions for the partial ordering generated by any orthant $K$ of $R^{n}$ to be preserved by a flow by Burton and Whyburn [7]. Historically, the motivation for these results was to obtain upper (maximal) and lower (minimal) solutions to a given

* Received by the editors July 22, 1985; accepted for publication (in revised form) August 21, 1986.

$\dagger$ Department of Mathematics, Arizona State University, Tempe, Arizona 85287. 
initial value problem, where typically $f$ was assumed only to be continuous. We refer the reader to one of the texts [10], [31], [59] for a fuller discussion of these results.

Our motivation in this paper is quite different. We assume enough smoothness on $f$ that (1.1) generates a unique solution for each initial condition in $U$ and that the solution depends smoothly on initial conditions. Our focus is on the asymptotic behavior of solutions of (1.1) and on the constraints on this limiting behavior imposed by the fact that the flow preserves a partial ordering. As mentioned, this paper consists of a survey of results on this subject, most of which are in the literature but which are not well known, at least to many in the applied areas, and most of which are quite recent. There would be no reason for such a survey if not for the impressive recent results of Hirsch [19]-[23] — there would be few results to survey. Indeed, the author's own contributions in this paper and in [46]-[48], [50], [51] have by and large been inspired by the work of Hirsch. However, there have been many other authors who have contributed important results. Among these, we mention Selgrade [40], [42], Martin [32], Matano [33] and Krasnosel'skii [28].

In the literature of applied mathematics, there are numerous examples of systems of differential equations for which the flow preserves a partial ordering. The gonorrhea model due to Lajmanovich and Yorke [30]

$$
y_{i}^{\prime}=-\alpha_{i} y_{i}-\left(c_{i}-y_{i}\right) \sum_{j=1}^{n} \beta_{i j} y_{j}, \quad 1 \leqq i \leqq n
$$

is an example of a system for which the flow preserves the usual partial ordering in the subset $\prod_{i=1}^{n}\left[0, c_{i}\right]$ of $R_{+}^{n}$. Under suitable conditions and when all constants are nonnegative, all solutions tend to zero in the feasible region or all nontrivial solutions tend to a unique positive equilibrium [30], [2]. Othmer [36] and Selgrade [40] have studied biochemical feedback loops modeled by the following system:

$$
\begin{aligned}
& x_{1}^{\prime}=f\left(x_{n}\right)-\alpha_{1} x_{1}, \\
& x_{i}^{\prime}=x_{i-1}-\alpha_{i} x_{i},
\end{aligned}
$$

The $x_{i}$ represent chemical concentrations so the natural domain is $R_{+}^{n}$. If $f^{\prime} \geqq 0$, the system describes a positive feedback loop and the usual ordering on $R^{n}$ is preserved by the flow. The set of steady states of the system, when nonempty, is typically a totally ordered subset with alternating stable and unstable steady states [40].

In population biology, one finds many examples of systems for which the flow preserves a partial order. For example, the two species Kolmogorov model of competition

$$
\begin{aligned}
& x^{\prime}=x f_{1}(x, y), \\
& y^{\prime}=y f_{2}(x, y), \quad(x, y) \in R_{+}^{2}
\end{aligned}
$$

in which $\partial f_{1} / \partial y \leqq 0$ and $\partial f_{2} / \partial x \leqq 0$ is an example of a system whose flow preserves the partial ordering generated by the second quadrant in $R^{2}$, the time reversed flow preserves the usual ordering. It is known [17], [24] that all bounded solutions of the system tend to a steady state. Takeuchi, Adachi and Tokumaru [56], [57] and Post, Travis and DeAngelis [38], [58] study the Lotka-Volterra system modeling competition between two subcommunities of species, each community consisting of species which act cooperatively among themselves. The system can be written as follows:

$$
\begin{aligned}
& x^{\prime}=\operatorname{diag}(x)\left[r_{1}+A x-B y\right], \\
& y^{\prime}=\operatorname{diag}(y)\left[r_{2}-C x+D y\right],
\end{aligned}
$$


in which $(x, y) \in R_{+}^{k} \times R_{+}^{n-k}, A$ is a $k \times k$ matrix whose off diagonal entries are nonnegative, $D$ is $(n-k) \times(n-k)$ with the same property and $B$ and $C$ have nonnegative entries. The components of $x$ denote the density of the species in one community and those of $y$ denote the densities of the other community. The flow of this system preserves the partial ordering generated by the cone $R_{+}^{k} \times\left(-R_{+}^{n-k}\right)$. Remarkable results for such systems have been obtained by Takeuchi and Adachi [56] and Travis and Post [58] without using the monotonicity properties of the flow. Some of these results rely on Lyapunov function arguments due to Goh [15], [16]. The author has exploited the monotonicity of the flow to study more general Kolmogorov type models of competition between subcommunities in [50], [51] making use of results of Hirsch and Selgrade.

Finally, we mention that systems (1.1) arise in the modeling of a dynamic market in which the prices of $n$ commodities form the components of $x$ and $f_{i}$ is proportional to the excess demand function. In case the various commodities are substitutable, then the flow of (1.1) will preserve the usual partial ordering on $R^{n}$ (see, e.g., [43]).

For further examples of systems with the property that the flow preserves a partial ordering for $t>0$ we recommend the book by De Angelis, Post and Travis [11] (see also §5).

While the present article is intended as a survey of results, it is by no means a complete one. We have selected results to be included here based solely on the bias of the author and on a desire to keep the paper to a manageable length. An important factor in deciding which results to include and which to exclude is the applicability of a particular result to concrete applications. As a result of this bias, many important results have been excluded. A reader interested in exploring this field to a greater depth should certainly consult the work of Hirsch [19]-[23], Selgrade [40]-[42], Matano [33] and Krasnosel'skii [28].

The organization of this paper is as follows. In $\$ 2$ we present necessary and sufficient conditions for the forward flow map $\phi_{t}$ to preserve a partial ordering generated by an orthant of $R^{n}$. Then we turn to the central problem, that of determining the asymptotic behavior of solutions of (1.1). Certainly the most important result, due to Hirsch [20, Thm. 2.5], says roughly that almost every solution of (1.1) tends to an equilibrium if a partial ordering (and a certain other condition) is preserved. It should be remarked that the results of Hirsch [20]-[23] were formulated for monotone flows in (possibly) infinite-dimensional spaces which are strongly ordered (see also Matano [33]). We will state these results as they apply to finitedimensional flows which preserve a partial ordering generated by an orthant. Simple results, due to Hirsch [19] and Selgrade [40], which give sufficient conditions for a particular solution to converge will be stated.

If most solutions of (1.1) tend to equilibria, then it becomes doubly important to find and determine the stability type of the various equilibria. For systems (1.1) whose flow preserves a partial ordering generated by an orthant, we find that $D f(x)$ is similar to a matrix having nonnegative off-diagonal elements. There is an extensive literature on such matrices which stems from the fact that the Perron-Frobenius theory of nonnegative matrices [13] applies to them. We refer the reader to the references [12], [13], [26], [37] and especially to [5]. It turns out that there is a simple necessary and sufficient test for $D f(x)$ to be a stable matrix (eigenvalues in the open left half-plane) which involves computing the signs of the principal minors of a matrix associated to $D f(x)$. This and other results from the linear algebra of these matrices are discussed in $\S 2$.

The behavior of solutions of (1.1) in the neighborhood of an unstable steady state is strongly influenced by the fact that a partial ordering is preserved by the flow. We 
show in $\$ 2$ that one typically expects monotone heteroclinic orbits to issue from unstable steady states and to terminate either at infinity or on a stable steady state. This result places strong constraints on the geometry of the set of equilibria of (1.1). In addition, we generalize a result of Selgrade [40], which gives geometrical information concerning the stable manifold of an unstable steady state. From these results there emerges a general description of the geometry of the set of equilibria which we discuss in $\S 2$.

Periodic orbits would seem to be of little importance for systems (1.1) preserving a partial ordering. Hirsch has observed that they can never be attracting [19]. However, periodic orbits are important for systems (1.1) for which the time reversed flow preserves a partial ordering, the so-called competitive systems, which can have attracting closed orbits [19], [52]. We do not consider competitive systems in this paper, although many of the results stated here can be applied to such systems by time reversal. We conclude $\$ 2$ with some brief remarks concerning periodic orbits.

In $\$ 3$ we describe a simple algorithm for identifying whether or not the flow corresponding to a particular system (1.1) preserves a partial ordering generated by an orthant. Briefly, the off-diagonal elements of $D f(x)$, the Jacobian matrix of $f$, should not change sign in $U$ (sign stability), $D f(x)$ should be sign symmetric $\left(\left(\partial f_{i} / \partial x_{j}\right)(x)\right.$ and $\left(\partial f_{j} / \partial x_{i}\right)(y)$ should have the same sign for $\left.x, y \in U, i \neq j\right)$ and, in the words of Travis and Post [58], "friends of friends are friends, friends of enemies are enemies and enemies of enemies are friends." The algorithm lends itself to a simple graph theoretic interpretation which we describe in $\$ 3$.

Armed with this algorithm, we describe certain classes of systems (1.1) possessing order preserving flows. We are led naturally to the class of tridiagonal monotone systems. This class was singled out by Smillie [45] who proved that all bounded semiorbits converge to equilibria. This result should have important applications.

In $\$ 4$ we indulge ourselves by describing some results which are somewhat outside the scope of the material in the previous sections. We discuss some very nice comparison results due to Conway and Smoller [9] and Gardner [14] for reaction diffusion systems:

$$
\begin{aligned}
& u_{t}=D \Delta u+f(u), \quad x \in \Omega, \\
& \frac{\partial u}{\partial v}=0 \quad \text { on } \partial \Omega, \\
& u(x, 0)=u^{0}(x), \quad x \in \Omega
\end{aligned}
$$

with Neumann boundary conditions. Here, $\Omega \subseteq R^{m}, u: \Omega \rightarrow R^{n}, D$ is a positive diagonal matrix and $v$ is an outer normal to $\partial \Omega$. It is not assumed that the reaction term $f$ possesses any monotonicity properties but only that $f$ points inward along the boundary of an $n$-dimensional rectangle. Under these, and additional assumptions, the above-mentioned authors associate $2^{n-1}$ pairs of autonomous ordinary differential systems which bound, with respect to various partial orderings, solutions of the reaction diffusion system for which $u^{0}(x)$ belongs to the rectangle for $x$ in $\Omega$.

It turns out that this result is most naturally stated in terms of the type $\mathrm{K}$ monotone systems introduced in $\S 2\left(\phi_{t}\right.$ preserves the partial ordering induced by the orthant $K$ ). Indeed, each of the $2^{n-1}$ pairs of comparison systems is a type $\mathrm{K}$ monotone system where $K$ runs through $2^{n-1}$ of the orthants of $R^{n}$, and these comparison systems have a certain natural characterization as minimal and maximal type $K$ monotone systems with respect to the reactive term $f$. These facts, together with the results of $\S 2$ give a clearer picture of what one can expect to accomplish by using the comparison 
technique. Perhaps the principle observation is that the comparison technique is ideal for the study of the stability of constant steady states of reaction diffusion systems for which the reactive term $f$ is a type $\mathrm{K}$ monotone system.

We mention that all the ideas in $\$ 4$ are applicable to ordinary differential systems (1.1) $(D=0)$ with $f$ satisfying the constraint mentioned above.

Finally, we should note that it is not our intent to survey monotonicity results for partial differential systems. This author is certainly unqualified for such a task. It should be pointed out, however, that many of the ideas and results described in $\$ 2$ have counterparts for nonlinear parabolic systems and in some cases it appears that these ideas were first applied to partial differential equations. Besides the results of Hirsch [20]-[23] which were intended for application to possibly infinite-dimensional systems, we should mention the work of Matano [33], Martin [32], Sattinger [39] and Kuiper [29].

In $\$ 5$ we present an application of the results of the previous sections to a mathematical model of repressible cyclic gene systems. We obtain results for general repressible terms which generalizes earlier work of Banks and Mahaffy [4] in the case that there are no time delays and the number of participating genes is even.

This author has extended many of the results discussed in this survey to apply to functional differential equations in [53].

We end this section with some notation and conventions to be used throughout this paper. The letter $n$ will be reserved for the dimension of $R^{n}$ and $N$ will denote the set $\{1,2,3, \cdots, n\}$. If $K$ is an orthant of $R^{n}$ we write $x \leqq_{K} y\left(x<<_{K} y\right)$ if and only if $y-x \in K(y-x \in$ interior of $K)$. If $x \leqq_{K} y$, let $[x, y]_{K}=\left\{z \in R^{n}: x \leqq_{K} z \leqq_{K} y\right\}$. The subscript $K$ will be dropped in case $K=R_{+}^{n}$, the nonnegative orthant.

2. Type K monotone systems. The purpose of the present section is to define the class of type $\mathrm{K}$ monotone systems and to describe some results which are known for such systems.

We begin by considering

$$
x^{\prime}=f(x)
$$

where $f$ is a continuously differentiable function defined on a convex, open set $U$ in $R^{n}$ (see Remark 3 below for a weakening of the convexity requirement). We seek sufficient conditions for the flow associated with (2.1) to preserve a partial ordering on $R^{n}$ generated by an orthant. More precisely, let $m=\left(m_{1}, \cdots, m_{n}\right), m_{i} \in\{0,1\}$, $1 \leqq i \leqq n$, and $K_{m}=\left\{x \in R^{n}:(-1)^{m_{i}} x_{i} \geqq 0,1 \leqq i \leqq n\right\}$. We say that the solution operator $\phi_{t}$ of (2.1) preserves the partial ordering $\leqq_{K}$ (for $\left.t \geqq 0\right)$ and (2.1) is type $K_{m}$ monotone if whenever $x, y \in U$ with $x \leqq_{K_{m}} y$ then $\phi_{t}(x) \leqq_{K_{m}} \phi_{t}(y)$ for all $t \geqq 0$ for which both $\phi_{t}(x)$ and $\phi_{t}(y)$ are defined. The following lemma gives necessary and sufficient conditions for (2.1) to be a type $K_{m}$ monotone system in the case that $f \in C^{1}(U)$ for an open convex set $U$ in $R^{n}$.

LEMMA 2.1. If $f \in C^{1}(U)$ where $U$ is open and convex in $R^{n}$ then $\phi_{t}$ preserves the partial ordering $\leqq_{K_{m}}$ for $t \geqq 0$ if and only if $P_{m} D f(x) P_{m}$ has nonnegative off-diagonal elements for every $x \in U$, where $P_{m}=\operatorname{diag}\left((-1)^{m_{1}}, \cdots,(-1)^{m_{n}}\right)$.

In order to simplify notation, we will hereafter drop the subscript $m$ on $P$ and on $K$.

Proof. (Sufficiency). Define $g: P U \rightarrow R^{n}$ by $g(x)=P f(P x)$. Then $D g(x)=$ $P D f(P x) P$ so $D g(x)$ has nonnegative off-diagonal elements and satisfies the Kamke condition [27], [35]. Hence $\phi_{t}^{g}(\cdot)$ preserves $\leqq$ for $t \geqq 0$. But $\phi_{t}^{f}(x)=P \phi_{t}^{g}(P x)$, so if $x \leqq_{K_{m}} y$ then $P x \leqq P y$, so $\phi_{t}^{g}(P x) \leqq \phi_{t}^{g}(P y)$, so $P \phi_{t}^{g}(P x) \leqq_{K_{m}} P \phi_{t}^{g}(P y)$ or $\phi_{t}^{f}(x) \leqq_{K_{m}} \phi_{t}^{f}(y)$. 
(Necessity). If $\phi_{t}(\cdot)=\phi_{t}^{f}(\cdot)$ preserves $K_{m}$ then since $x \leqq_{K_{m}} x+(-1)^{m_{j}} s e_{j}$ for $s \geqq 0$, we have

$$
\phi_{t}(x) \varliminf_{K_{m}} \phi_{t}\left(x+(-1)^{m_{j}} s e_{j}\right) \quad \text { for } t \geqq 0, \quad s \geqq 0
$$

or

$$
0 \leqq_{K_{m}} \frac{\phi_{t}\left(x+(-1)^{m_{j}} s e_{j}\right)-\phi_{t}(x)}{s}, \quad s>0, \quad t \geqq 0
$$

so

$$
0 \leqq_{K_{m}}(-1)^{m_{j}} \frac{\partial \phi_{t}}{\partial x_{j}}(x)
$$

so

$$
0 \leqq(-1)^{m_{j}+m_{i}} \frac{\partial \phi_{t}^{i}}{\partial x_{j}}(x), \quad t \geqq 0
$$

so

$$
P \frac{\partial \phi_{t}}{\partial x}(x) P \geqq 0, \quad P=\operatorname{diag}\left((-1)^{m_{1}}, \cdots,(-1)^{m_{n}}\right) .
$$

Since $\left(\partial \phi_{0} / \partial x\right)(x)=I$ it follows that

$$
\left.\frac{\partial}{\partial t}\right|_{t=0}\left[P \frac{\partial \phi_{t}}{\partial x}(x) P\right]_{i j} \geqq 0 \quad \text { if } i \neq j .
$$

But

$$
\left.\frac{\partial}{\partial t}\right|_{t=0}\left[\frac{\partial \phi_{t}}{\partial x}(x)\right]=D f(x)
$$

So

$$
\left.\frac{\partial}{\partial t}\right|_{t=0} P \frac{\partial \phi_{t}}{\partial x}(x) P=P D f(x) P
$$

so $(P D f(x) P)_{i j} \geqq 0$ if $i \neq j$. This completes our proof.

Remark 1. The proof shows that if $(2.1)$ is type $\mathrm{K}$ monotone, then the change of variables $y=P x$ in (2.1) yields a system $y^{\prime}=g(y), g \in C^{1}(P U), g(y)=P f(P y)$, for which the flow $\psi_{t}$ preserves the usual partial ordering $\leqq$ on $R^{n}$ and $D g(y)$ has nonnegative off-diagonal elements in $P U$. Thus, when it is useful to do so, we will feel free to establish our future results for this case only. In the applications, however, one often has some intuition concerning the system (2.1) which may be obscured by making the above change of variables.

A "canonical" form for type $\mathrm{K}$ monotone systems can be obtained by permuting equations and variables in (2.1) in the same way so that $m$ consists of $k$ ones followed by $n-k$ minus ones. In this way we arrive at a system (2.1) where $x=\left(x_{1}, x_{2}\right) \in R^{k} \times R^{n-k}, f=\left(f_{1}, f_{2}\right)$ and

$$
D f(x)=\left[\begin{array}{l:c}
\frac{\partial f_{1}}{\partial x_{1}} & \frac{\partial f_{1}}{\partial x_{2}} \\
\hdashline \frac{\partial f_{2}}{\partial x_{1}} & \frac{\partial f_{2}}{\partial x_{2}}
\end{array}\right]
$$


where $\partial f_{1} / \partial x_{1}$ and $\partial f_{2} / \partial x_{2}$ have nonnegative off-diagonal elements and $\partial f_{1} / \partial x_{2} \leqq 0$, $\partial f_{2} / \partial x_{1} \leqq 0$. In these coordinates, the flow of $(2.1)$ preserves the ordering induced by the cone $R_{+}^{k} \times\left(-R_{+}^{n-k}\right)$.

Remark 2. The proof shows that (2.1) is type $\mathrm{K}$ monotone if and only if

$$
P \frac{\partial \phi_{t}}{\partial x}(x) P \geqq 0, \quad t \geqq 0, \quad x \in U .
$$

Remark 3. The requirement of convexity of $U$ can be weakened. As pointed out by Hirsch [19], it suffices for $U$ to be "order" convex in the sense that if $x, y \in U$ with $x \leqq_{K} y$ then $t x+(1-t) y \in U$ for $0 \leqq t \leqq 1$. Of course, $\phi_{t}$ will preserve $\leqq_{K}$ for $t \geqq 0$ under weaker conditions than those described in Lemma 2.1. For example, if $f$ is locally Lipschitz in $U$ and for each $i, f_{i}$ satisfies the condition that whenever $x$ and $y$ are in $U$ with $x_{i}=y_{i}$ and $x_{j} \leqq y_{j}, j \neq i$, it follows that $f_{i}(x) \leqq f_{i}(y)$, then $\phi_{t}$ preserves the usual ordering $\leqq$ for $t \geqq 0$. This approach can be modified to consider the preservation of $\varliminf_{K}$ as well (see, e.g., [7], [31], [51] and (H) of §4). In this way we could avoid restrictions on the set $U$ and weaken our smoothness requirements on $f$.

Remark 4. Lemma 2.1 leads to a useful algorithm for testing a system (2.1) in order to see if it is type $\mathrm{K}$ monotone for some $m$ (and for finding $m$ ). Although for small values of $n$, it is usually a trivial matter to identify a type $\mathrm{K}$ monotone system, when $n$ is large it can be a nontrivial matter. We will describe a simple algorithm for detecting type $\mathrm{K}$ monotonicity in the next section. Here, we content ourselves by reinterpreting the sufficient condition of Lemma 2.1 as

$$
(-1)^{m_{i}+m_{j}} \frac{\partial f_{i}}{\partial x_{j}}(x) \geqq 0, \quad i \neq j, \quad x \in U
$$

and noting that it requires "sign stability" of the off-diagonal elements of $D f(x)$, i.e., $\partial f_{i} / \partial x_{j}$ cannot have opposite signs in $U$ for each $i, j, i \neq j$, and it requires "sign symmetry" of $D f(x)$ in the sense that $\left(\partial f_{i} / \partial x_{j}\right)\left(\partial f_{j} / \partial x_{i}\right) \geqq 0$ in $U$. These conditions are not sufficient however (see $\$ 3$ ).

A significantly stronger monotonicity property is enjoyed by the flow $\phi_{t}$ in the case that $D f(x)$ is an irreducible matrix for each $x \in U$. We say (2.1) is irreducible in this case. Recall that an $n \times n$ matrix is irreducible if it does not leave invariant any proper nontrivial subspace generated by a subset of the standard basis vectors for $R^{n}$ or, equivalently, if one cannot put the matrix in the following form:

$$
\left[\begin{array}{c:c}
\mathrm{A} & \mathrm{B} \\
\hdashline 0 & \mathrm{C}
\end{array}\right]
$$

where $\mathrm{A}$ and $\mathrm{C}$ are square matrices by a reordering of the standard basis (see [13]). Somewhat inaccurately, (2.1) is irreducible if it cannot be decomposed into two subsystems, one of which does not depend on the other (imagine a linear system with the above coefficient matrix). The result below has been proved by a number of authors in varying degrees of generality (see Berman and Plemmons [5], Martin [32], Aronsson and Kellogg [1] and Hirsch [20]). Hirsch [20] appears to be the first to fully exploit the hypothesis of irreducibility.

LEMMA 2.2. If (2.1) is an irreducible type $K$ monotone system then $P\left(\partial \phi_{t} / \partial x\right)(x) P>0$ for $t>0$. Hence, if $x, y$ are distinct points of $U$ with $x \leqq_{K} y$ then $\phi_{t}(x) \leqq_{K} \phi_{t}(y)$ for $t>0$.

A proof of Lemma 2.2 can be found in Hirsch [20]. We note that the second assertion of the lemma follows immediately from the first together with the 
fundamental theorem of calculus. The stronger monotonicity property enjoyed by irreducible type $\mathrm{K}$ monotone systems has the following noteworthy corollary: If $x$ and $y$ are distinct equilibria with $x \leqq y$, then in fact $x<_{K} y$.

In addition to the stronger monotonicity property enjoyed by irreducible type $\mathrm{K}$ monotone systems, there is another advantage of the irreducibility hypothesis. By Lemma 2.1 , if $(2.1)$ is type $\mathrm{K}$ monotone and $t \geqq 0$ then $P\left(\partial \phi_{t} / \partial x\right) P \geqq 0$ and by Lemma 2.2, if in addition (2.1) is irreducible and $t>0$, then the matrix is positive (all elements are positive). The Perron-Frobenius theorem yields stronger results for positive matrices than for nonnegative matrices [13], [5]. Recall that for a nonnegative matrix $\mathbf{A}$, the spectral radius of $\mathbf{A}$ is an eigenvalue and there is a corresponding nonnegative eigenvector. If $\mathbf{A}$ is a positive matrix then the spectral radius must be a positive simple eigenvalue strictly larger in modulus than all remaining eigenvalues and the corresponding eigenvector is positive and the only nonnegative eigenvector up to scalar multiple. These stronger spectral properties of positive matrices can be exploited in a differential equations setting (see [52] and later remarks in this section).

The asymptotic behavior of a typical solution of a type $\mathrm{K}$ monotone system is convergence to equilibrium. This statement is more precisely formulated in a result of Hirsch which we will describe in a later paragraph. Our immediate goal is to describe two relatively simple sufficient conditions for a bounded solution to converge to an equilibrium.

LEMMA 2.3. Let $O^{+}(x)=\left\{\phi_{t}(x): t \geqq 0\right\}$ be a semi-orbit of a type $K$ monotone system and suppose $\mathrm{O}^{+}(x)$ has compact closure in $U$. Then either of the following is sufficient for $\phi_{t}(x)$ to tend to an equilibrium as $t \rightarrow \infty$ :

(a) (Selgrade [41]) $f(x) \geqq_{K} 0\left(f(x) \leqq_{K} 0\right)$,

(b) (Hirsch [19]) $\phi_{T}(x)>_{K} x\left(\phi_{T}(x)<_{K} x\right)$ for some $T>0$.

Both (a) and (b) are extremely useful, (a) being more useful in the applications while (b) is a fundamental theoretical tool. We give a brief informal sketch of the proof. In the case of (a) with $f(x) \geqq_{K} 0$, it is immediate from the monotonicity properties of $f$ that $x+K_{m}$ is positively invariant for (2.1) (it is easiest, and it suffices, to see this for $\left.K_{m}=R_{+}^{n}\right)$. Hence $\phi_{t}(x) \geqq_{K_{m}} x$ for $t \geqq 0$ and thus $\phi_{t+s}(x) \geqq_{K_{m}} \phi_{s}(x)$ whenever $s \geqq 0$ by the monotonicity of $\phi_{s}$. It follows that the components of $\phi_{t}(x)$ are monotone functions of $t$ and this proves (a). In case (b) with $\phi_{T}(x)>_{K} x$, it follows that $\lim _{n \rightarrow \infty} \phi_{n T}(x)=\bar{x}$ exists since the components of $\left\{\phi_{n T}(x)\right\}_{n \geqq 0}$ are monotone in $n$ and bounded. By continuity, $\phi_{T}(\bar{x})=\bar{x}$, so $O(\bar{x})=\left\{\phi_{t}(\bar{x}): t \in R\right\}$ is a closed orbit having $T$ as a period and the omega limit set of $x$ is $O(\bar{x})$. Since the inequality $\phi_{T}(x)>_{K} x$ is a strict one, $\phi_{T^{\prime}}(x)>x$ for $T^{\prime}$ in an open set containing $T$. Thus the closed orbit $O(\bar{x})$ must have a nonempty open set of periods. It follows that $O(\bar{x})$ is an equilibrium.

For examples of the application of (a) to concrete systems the reader is referred to [40], [51]. Hirsch [19] observes numerous consequences of (b). Noteworthy among these are that a type K-monotone system cannot have an attracting closed orbit nor can any two points of an omega limit set be related by $<_{K}$. For in either case, one can find an appropriate orbit satisfying (b) and providing an appropriate contradiction.

In low dimensions $(n \leqq 3)$ the $\alpha$ and $\omega$ limit sets of a type K-monotone system can be completely categorized. In two dimensions, a bounded positive or negative semiorbit must converge to equilibrium in an eventually monotone manner. This fact has been noted by several authors [17], [23], [40]. In three dimensions we have for type K-monotone systems.

THEOREM 2.4 (Hirsch [19]). If $n=3$ and $L$ is an omega limit set which contains no equilibria then $L$ is a (nonattracting) closed orbit. If $L$ is an alpha limit set containing no equilibria then $L$ is either a closed orbit or a cylinder of closed orbits. 
This author showed in [52] that an alpha limit set containing no equilibria must be a closed orbit if the system is an irreducible type $\mathrm{K}$ monotone system (see [52] for other conditions implying this result). This result is most important for so-called competitive systems for which a partial ordering is preserved for $\phi_{t}, \mathrm{t} \leqq 0$. Such systems can have attracting closed obits [52].

For $n>3$, no such general result exists. In fact, it is known that type K-monotone systems can possess invariant sets on which the flow is as complicated as for invariant sets of arbitrary smooth systems. Smale [44] has proved that any smooth vector field on the standard $n-1$ simplex in $R^{n}$ can be extended to smooth vector field on $R^{n}$ which is type $\mathrm{K}$ monotone $\left(K=R_{+}^{n}\right)$ in $R_{+}^{n}$. Although the simplex is a repeller in this construction, the dynamics on the invariant simplex may be arbitrarily complex. Hirsch [19] has proved a sort of converse to this result of Smale implying that a limit set of a type $\mathrm{K}$ monotone system can be no more complicated than an invariant set for a general system in one less dimension. We will not give a precise statement of this result. Instead, we state the following.

THEOREM 2.5 (Hirsch [20], [21]). Let $\phi_{t}$ be the flow of an irreducible type $K$ monotone system with the property that all forward orbits have compact closures. Assume that the set of steady states of (2.1) is a discrete set in $U$. Then the set $Y$ of points $x \in U$ for which $\phi_{t}(x)$ does not converge to a steady state of (2.1) has Lebesgue measure zero.

We have strengthened considerably the hypotheses used by Hirsch [20, Thm. 4.1] in order to avoid some definitions and a somewhat clumsier statement. Theorem 2.5 is an immediate consequence of a sharper result of Hirsch which we will have occasion to use later in this section. This result asserts that if $\phi_{t}$ is the flow of an irreducible type $\mathrm{K}$ monotone system and if $S$ is a nonempty simply ordered subset of $U$ containing points whose forward orbits are bounded, then the subset $S_{0}$ of points of $S$ whose positive limit sets do not belong to the set $E$ of equilibria is countable.

A much stronger assertion than that of Theorem 2.5 can be made in the case that more is assumed about the properties of the flow. Let $E$ denote the set of steady states of (2.1). If $e \in E$, let $B(e)$ denote the basin of attraction of $e$. The following result is a consequence of Theorem 9.4 of Hirsch [23].

THEOREM 2.6. Let $X_{0}$ be an open, bounded, positively invariant set for an irreducible type $K$ monotone system, whose closure, $\bar{X}_{0}$, contains a finite number of equilibria. Then

$$
\bigcup_{e \in E \cap \bar{X}_{0}} \operatorname{Int}(B(e))
$$

is open and dense in $X_{0}$.

Int $(S)$ denotes the interior of $S$. It is not difficult to see that $\operatorname{Int}(B(e))=\varnothing$ if $e$ is linearly unstable (there exists an eigenvalue of the Jacobian with positive real part).

The results of Hirsch suggest that the asymptotic behavior of a typical solution of a type $\mathrm{K}$ monotone system is convergence to an equilibrium. Thus it will be particularly important to determine the stability type of each equilibrium of (2.1). If $x_{0}$ is an equilibrium of (2.1) then the matrix $A=D f\left(x_{o}\right)$ is the coefficient matrix of the variational equation about $x_{0}$. By Lemma $2.1, A$ is similar to a matrix whose offdiagonal elements are nonnegative. We will call any matrix $A$ having the property that the off-diagonal elements of $P_{m} A P_{m}$ are nonnegative for some $m$, a type $\mathrm{K}$ matrix. We write $s(A)$ for the stability modulus of an $\mathrm{n} \times \mathrm{n}$ matrix, $s(A)=\max \operatorname{Re} \lambda$ where $\lambda$ runs over the spectrum of $A$. We call $A$ a stable matrix if $s(A)<0$. The following result summarizes some of the special properties of type $\mathrm{K}$ matrices. 
Theorem 2.7. Let $A$ be a type $K$ matrix. Then $s(A)$ is an eigenvalue of $A$ and there exists a corresponding eigenvector $v \in K$. The following are equivalent:

(i) $s(A)<0$.

(ii) There exists $u>_{K} 0$ such that $A u<_{K} 0$.

(iii) $-A^{-1} \geqq_{K} 0$.

(iv)

$$
(-1)^{k}\left|\begin{array}{cccc}
a_{11} & \left|a_{12}\right| & \ldots & \left|a_{1 k}\right| \\
\left|a_{21}\right| & a_{22} & \left|a_{23}\right| & \left|a_{2 k}\right| \\
\vdots & & & \vdots \\
\left|a_{k 1}\right| & \ldots & & \left|a_{k, k-1}\right| a_{k k}
\end{array}\right|>0, \quad 1 \leqq k \leqq n .
$$

(v) There exists $d>0$ such that the symmetric matrix $\operatorname{diag}(d) A+A^{t} \operatorname{diag}(d)$ is negative definite, where diag $(d)$ is the diagonal matrix with $d_{1}, \cdots, d_{n}$ down the main diagonal.

We remark that the inequality $B \geqq_{K} 0$ for an $n \times n$ matrix means precisely that $B$ maps the orthant $K$ into itself.

The reader may have guessed that the Perron-Frobenius theorem lies behind many of the assertions of Theorem 2.7. For a proof of most of Theorem 2.7 and for many other interesting facts about type $\mathrm{K}$ matrices, we refer the reader to Berman and Plemmons [5, Chap. 6] where they are called $M$ matrices (see also Fiedler and Ptak [12] and Johnson [26]).

In order to interpret the results of Theorem 2.7 it is useful, and causes no loss of generality, to assume that $A$ has nonnegative off-diagonal elements, in which case the partial ordering is the usual one. Notice that by (ii), $A$ can be stable only if each diagonal element is negative and dominates the other entries in that row. This result is certainly intuitive. It is quite remarkable that (iv) is precisely the test for negative definiteness of a symmetric matrix (ignoring the absolute values in the general case). We have a straightforward test for stability of a type $\mathrm{K}$ monotone system, in contrast to the case of a general matrix. The various other equivalent conditions have proved to be useful and have been exploited by many authors [15], [16], [30], [38], [40], [43], [50], [51], [57], [58]. For example (v) has been used by Goh [15], [16] to obtain Lyapunov functions for Lotka-Volterra equations (see also [56], [57]). The equivalent condition (ii) has a simple geometrical interpretation for a steady state $x_{0}$ of (2.1) where $A=D f\left(x_{0}\right)$. It implies that

$$
(-1)^{\sigma} f\left(x_{0}+(-1)^{\sigma} t u\right)<{ }_{K} 0, \quad \sigma=0,1
$$

for sufficiently small positive $t$. It follows from the monotonicity of $f$ that the "interval" $\left[x_{0}-t u, x_{0}+t u\right]_{K}$ is positively invariant for (2.1). Indeed, the vector field, on the boundary, points into $\left[x_{0}-t u, x_{0}+t u\right]_{K}$ for small positive $t$.

It is interesting to consider the bifurcations which can occur from a steady state of a type $\mathrm{K}$ monotone system in view of Theorem 2.7. It is not difficult to see from the Perron-Frobenius theorem that if $\lambda=\alpha+i \beta, \beta \neq 0$ is an eigenvalue of $A=D f\left(x_{o}\right)$ then $\alpha<s(A)$. It follows that a stable steady state loses stability as a parameter is varied by a real eigenvalue crossing from negative to positive on the real axis. A complex conjugate pair of eigenvalues can cross the imaginary axis only after a real eigenvalue has changed sign. Hence a locally stable Hopf bifurcation cannot occur for type $\mathrm{K}$ monotone systems. Unstable Hopf bifurcations can occur from unstable steady states as shown by Selgrade [41]. The Perron-Frobenius theorem also implies that $s(A)$ is a simple eigenvalue in case $\mathrm{A}$ and is an irreducible type $\mathrm{K}$ matrix. It is 
well known that bifurcation of a steady state must always occur as a simple real eigenvalue crosses the imaginary axis. We see that the only local bifurcation involving an exchange of stability that a steady state of an irreducible type $\mathrm{K}$ monotone system can be involved in is a steady state bifurcation.

The existence of heteroclinic trajectories connecting steady states of dynamical systems with monotonicity properties has been considered by several authors. We mention results of Selgrade [42] and Matano [33], the latter results apply to a reaction diffusion equation as well. We present a result below which, while requiring more smoothness than the results of Selgrade and Matano, has the advantage that we need only suppose the existence of a single unstable steady state.

THEOREM 2.8. Suppose (2.1) is a type K monotone system and let fhave a locally Lipschitzian derivative in $U$. Suppose $f\left(x_{0}\right)=0, s_{0}=s\left(D f\left(x_{0}\right)\right)>0$ is a simple eigenvalue of $D f\left(x_{0}\right)$ with corresponding eigenvector $v \geqq_{K} 0$. Suppose $x_{0}+K$ is contained in $U$. Then there exists a unique $C^{1}$ function $y:[0, \infty) \rightarrow x_{0}+K$ with the following properties:

(1) $y(r)=x_{0}+r v+o(r)$ as $r \rightarrow 0$.

(2) $\phi_{t}(y(r))=y\left(e^{s_{0} t} r\right), t \in R, r \geqq 0$.

(3) $0 \leqq r_{1}<r_{2}$ implies $y\left(r_{1}\right) \leqq_{K} y\left(r_{2}\right)$.

(4) Either $\lim _{r \rightarrow \infty}\|y(r)\|=\infty$ or $\lim _{r \rightarrow \infty} y(r)=x_{1}$ where $x_{1} \geqq_{K} x_{0}, f\left(x_{1}\right)=0$. If $x_{1}>_{K} x_{0}$ then $s\left(D f\left(x_{1}\right)\right) \leqq 0$. If $D f\left(x_{1}\right)$ is irreducible and $s_{1}=s\left(D f\left(x_{1}\right)\right)$ with corresponding eigenvector $w \geqq_{K} 0$ then $\lim _{r \rightarrow \infty}\left(y^{\prime}(r) /\left\|y^{\prime}(r)\right\|\right)=w$.

(5) If $\lim _{r \rightarrow \infty}\|y(r)\|=\infty$ then $\left\|\phi_{t}(x)\right\| \rightarrow \infty$ as $t \rightarrow \infty$ for all $x \geqq_{K} x_{0}, x \neq x_{0}$. If $\lim _{r \rightarrow \infty} y(r)=x_{1}$ then $\phi_{t}(x) \rightarrow x_{1}$ for all $x$ with $x_{0} \leqq_{K} x \leqq_{K} x_{1}, x \neq x_{0}$.

Theorem 2.8 states that the monotone curve $\Gamma=\{y(r): r \geqq 0\}$ is a heteroclinic orbit of (2.1) connecting the unstable steady state $x_{0}$ to the steady state $x_{1}($ or $\infty)$. In addition, the steady state $x_{1}$, if not asymptotically stable, at least attracts all initial conditions $x$ with $x_{0} \leqq_{K} x \leqq_{K} x_{1}$. If $x_{1}>_{K} x_{0}$, then the domain of attraction of $x_{1}$ includes an open set so we may expect that, generically, $x_{1}$ is asymptotically stable $\left(s\left(D f\left(x_{1}\right)\right)<0\right)$. If $D f\left(x_{0}\right)$ is irreducible, then $v>_{K} 0$ and it follows from (1) Theorem 2.8 that $x_{1}>_{K} x_{0}$.

The functional identity (2) can be interpreted in terms of the following commutative diagram of mappings:

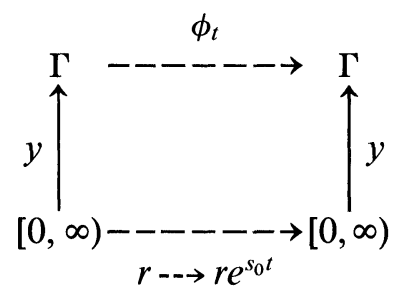

The parametrization $y$ of the heteroclinic orbit $\Gamma$ has been chosen in order to linearize the action of $\phi_{t}$ on $\Gamma$.

In Theorem 2.8, we have only stated half of the story. If $x_{0}-K$ is contained in $U$, there exists a unique $C^{1}$ function $z:[0, \infty) \rightarrow x_{0}-K$ satisfying $z(r)=x_{0}-r v+o(r)$ as $r \rightarrow 0$, (2) with $z$ replacing $y$, (3) $0 \leqq r_{1}<r_{2}$ implies $z\left(r_{1}\right) \geqq_{K} z\left(r_{2}\right)$, (4) with $x_{1} \leqq_{K} x_{0}$ and $\lim _{r \rightarrow \infty}\left(z^{\prime}(r) /\left\|z^{\prime}(r)\right\|\right)=-w$, and the obvious changes in (5).

The assumption that $x_{0}+K$ is contained in $U$ can be significantly weakened. It suffices for there to exist a closed positively invariant set $P$ for (2.1) in $U$ containing $x_{0}+r v$ for small positive $r$. In this case $y(r)$ lies in $x_{0}+K \cap P$ for $r \geqq 0$ (see [49, Remark 4]). 
In the generic case then, Theorem 2.8 implies that a steady state of $(2.1)$ is either asymptotically stable or there are two monotone heteroclinic orbits of $(2.1)$ connecting the steady state to two different asymptotically stable steady states.

Proof of Theorem 2.8. Theorem 2.8 was proved for mappings by the author in [49; see Thms. 1.1, 2.1, 2.2 and Remarks]. The translation from mappings to flows is fairly standard so we will be brief. Fix $t_{0}>0$ and note that the results of [49] apply to the map $T=\phi_{t_{0}}(\cdot)$; that is, $\phi_{t_{0}}\left(x_{0}\right)=x_{0}, D \phi_{t_{0}}\left(x_{0}\right) \geqq_{K} 0$ and $D \phi_{t_{0}}\left(x_{0}\right)$ has spectral radius $e^{s t_{0}}>1$ with corresponding eigenvector $v \geqq_{K} 0$. Theorems 1.1 and 2.1 of [49] apply to $T$ and we obtain a function $y_{t_{0}}:[0, \infty) \rightarrow x_{0}+K$ satisfying (1), (2) with $t$ replaced by $t_{0},(3),(4)$ except that $\phi_{t_{0}}\left(x_{1}\right)=x_{1}$ and $\rho\left(D \phi_{t_{0}}\left(x_{1}\right)\right) \leqq 1$ if $x_{1}>_{K} x_{0}$, and (5) except that $\left\|\phi_{p t_{0}}(x)\right\| \rightarrow \infty$ if $\lim _{r \rightarrow \infty}\left\|y_{t_{0}}(r)\right\|=\infty$ and $\phi_{p t_{0}}(x) \rightarrow x_{1}$ if $\lim _{r \rightarrow \infty} y_{t_{0}}(r)=x_{1}$, as $p \rightarrow \infty$. Also, by Theorem 1.1 in [49] we have $y_{t_{0}}(r)=\lim _{p \rightarrow \infty} \phi_{p t_{0}}\left(x_{0}+e^{-p s t_{0}} r v\right)$, the limit being uniform on compact $r$-sets.

Replacing $t_{0}$ above by $(p / q) t_{0}$ where $p$ and $q$ are positive integers we obtain $y_{(p / q) t_{0}}$ with properties as above and it is easy to see that $y_{(p / q) t_{0}}(r) \equiv y_{t_{0}}(r)$ on $[0, \infty)$. If $Q^{+}$ denotes the positive rational numbers then we have for $\eta, \omega \in Q^{+} t_{0}$ that

$$
\phi_{\eta}\left(y_{\omega}(r)\right)=\phi_{\eta}\left(y_{\eta}(r)\right)=y_{\eta}\left(e^{s \eta} r\right)=y_{\omega}\left(e^{s \eta} r\right) \text {, }
$$

the second equality following from (ii) of Theorem 1.1 in [49]. The equality $y_{\eta}(r)=\phi_{\eta}\left(y_{\omega}\left(r e^{-s \eta}\right)\right)$ from above can be used together with the uniqueness of $y_{t}$ for $t>0$ from Theorem 1.1 of [49] to show that the equality extends to all $\eta>0$ with $\omega \in Q^{+} t_{0}$. From this the continuity of the map $(\eta, r) \rightarrow y_{\eta}(r)$ follows and hence $y_{t_{1}}(r)=y_{t_{0}}(r)$ for any $t_{1}>0$. Now $\phi_{t}\left(y_{t_{0}}(r)\right)=\phi_{t}\left(y_{t}(r)\right)=y_{t}\left(e^{s t} r\right)$ so that (2) follows for $t>0$ where $y(r) \equiv y_{t_{0}}(r)$. Since (2) holds for $t>0$ it also holds for $t<0$ by applying $\phi_{t}$ to (2) with $t$ replaced by $-t$.

Since $\phi_{t}\left(x_{1}\right)=\lim _{r \rightarrow \infty} \phi_{t}(y(r))=\lim _{r \rightarrow \infty} \phi_{t}\left(y_{t}(r)\right)=\lim _{r \rightarrow \infty} y_{t}\left(e^{s t} r\right)=\lim _{r \rightarrow \infty} y\left(e^{s t} r\right)$ $=x_{1}$, it follows that $x_{1}$ is a steady state of $(2.1)$.

The remaining assertions are trivial to check using the monotonicity of $\phi_{t}$.

From Theorem 2.8 and the remarks following it, we conclude that an unstable steady state $x_{0}$ should give rise to two other steady states $x_{-1}$ and $x_{1}$ with $x_{-1} \leqq_{K} x_{0} \leqq_{K} x_{1}$ and we may expect, generically, that $x_{-1}$ and $x_{1}$ are asymptotically stable. The converse is also true [51, Lemma 3.7].

Proposition 2.9. Suppose $x_{1}$ and $x_{2}$ are steady states of $(2.1)$ with $s\left(D f\left(x_{i}\right)\right)<0$ for $i=1,2$ and $x_{1}<_{K} x_{2}$. Then there exists at least one steady state $x_{0}$ in $\left[x_{1}, x_{2}\right]_{K}$ with $s\left(D f\left(x_{0}\right)\right) \geqq 0$.

If (2.1) is an irreducible type $\mathrm{K}$ monotone system, one can make some generic statements about the set $E$ of equilibria based on the previous results. Recall that irreducibility implies that two steady states are either unrelated by $\leqq_{K}$ or are strongly related $\left(\right.$ by $\left.<_{K}\right)$. Assume that all equilibria are nondegenerate. Let $x_{1}$ and $x_{2}$ be two equilibria as in Proposition 2.9 and assume that there are no other stable equilibria in $\left[x_{1}, x_{2}\right]$ (if there are, replace for example $x_{2}$ by a "nearest to $x_{1}$ " stable equilibria). Then a simple Brouwer degree argument as in [51] shows that $\left[x_{1}, x_{2}\right]$ must contain, apart from $x_{1}$ and $x_{2}$, an odd number of equilibria and each of these must be unstable $(s>0)$. Theorem 2.8 implies that each of these unstable equilibria gives rise to a most unstable manifold connecting it to $x_{1}$ and to $x_{2}$. Such a block $\left[x_{1}, x_{2}\right]$ together with its unstable equilibria and connecting orbits can be viewed as a typical "atom" making up the set $E$.

Our final result concerns the geometry of the stable manifold of an unstable, hyperbolic, steady state of (2.1). If $x_{0}$ is such a steady state, we denote by $W^{s}\left(x_{0}\right)$ the stable manifold of $x_{0}$, a $C^{1}$ manifold, immersed in $U$, consisting of points which are 
asymptotic to $x_{0}$ as $t \rightarrow \infty$ for the flow. The content of Theorem 2.10 is that no two distinct points of $W^{s}\left(x_{0}\right)$ can be related by $\leqq_{K}$. Such a result was proved by Selgrade [40] in the special case that $K=R_{+}^{n}$ and $\operatorname{dim} W^{s}\left(x_{0}\right)=n-1$ for a particular class of equations. Theorem 2.10 extends his result.

THEOREM 2.10. Let (2.1) be a type K monotone system. Suppose $x_{0}$ is a hyperbolic steady state of $(2.1)$ where $s\left(D f\left(x_{0}\right)\right)>0$ and $D f\left(x_{0}\right)$ is irreducible. Then $W^{s}\left(x_{0}\right)$ does not contain distinct points $x$ and $y$ with $x \leqq_{K} y$.

Proof. We first show that the result holds for linear systems. By making the change of variables $y=P x$ where $P=\operatorname{diag}(1,1, \cdots, 1,-1,-1, \cdots,-1)$ has $k$ ones followed by $n-k$ minus ones down the diagonal, we may as well assume $K=R_{+}^{n}$ (see Remark 1 following Lemma 2.1). Taking $x_{0}=0$ and (2.1) as $x^{\prime}=A x$ where $a_{i j} \geqq 0$ if $i \neq j$ and $A$ irreducible, we have $e^{A t}>0$ by [1]. Thus if $u \in W^{s}(0)-\{0\}$ and $u \geqq 0$, then $e^{A t} u>0$ for $t>0$. But then there exists $v>0$ with $v \in W^{s}(0)$ (take $v=e^{A t} u$ for some $t>0)$. It follows that the set $\{x: 0 \leqq x \leqq v\}$ is contained in $W^{s}(0)$. Since the latter set has a nonempty interior we have a contradiction. Hence the result follows for linear type $\mathrm{K}$ monotone systems.

Now suppose the system is nonlinear and without loss of generality that $x_{0}=0$. Let $E^{s}$ denote the direct sum of the eigenspaces corresponding to eigenvalues of $D f(0)$ with negative real part. From the first paragraph we know that $E^{s}$ contains no two distinct points $x$ and $y$ with $x \leqq_{K} y$. There exists a neighborhood $U$ of zero such that $U \cap W^{s}(0)$ is positively invariant for (2.1) and such that there exists a neighborhood $V$ of 0 in $E^{s}$, a continuously differentiable map $h: V \rightarrow E^{c}$ where $E^{c}$ is a complementary subspace to $E^{s}$ in $R^{n}, R^{n}=E^{s}+E^{c},\|\cdot\|=\|\cdot\|_{1}+\|\cdot\|_{2}$, with $h(0)=0, D h(0)=0$ and $U \cap W^{s}(0)=\{(v, w): w=h(v), v \in V\}$. We now show that there exists a neighborhood $U^{1}$ of zero, $U^{1} \subset U$, such that no two distinct points of $U^{1} \cap W_{s}(0)$ are related by $\leqq_{K}$. If this were false then we could find two sequences of points $x_{p}$ and $y_{p}$ with $x_{p} \leqq_{K} y_{p}, x_{p} \neq y_{p}, x_{p}, y_{p} \in U \cap W_{s}(0)$ and $x_{p} \rightarrow 0, y_{p} \rightarrow 0$ as $p \rightarrow \infty$. Write $x_{p}=\left(v_{p}, h\left(v_{p}\right)\right), v_{p} \in V$ and $y_{p}=\left(\bar{v}_{p}, h\left(\bar{v}_{p}\right)\right), \bar{v}_{p} \in V$. Then $v_{p}, \bar{v}_{p} \rightarrow 0$ as $p \rightarrow \infty$ and

$$
\left(\frac{\bar{v}_{p}-v_{p}}{\left\|\bar{v}_{p}-v_{p}\right\|_{1}}, \frac{h\left(\bar{v}_{p}\right)-h\left(v_{p}\right)}{\left\|\bar{v}_{p}-v_{p}\right\|_{1}}\right) \geqq_{K} 0
$$

for $p=1,2, \cdots$. Now let $p \rightarrow \infty$ along a suitable subsequence for which $\left(\bar{v}_{p}-v_{p}\right) /\left(\left\|\bar{v}_{p}-v_{p}\right\|_{1}\right) \rightarrow e$ obtaining $(e, 0) \geqq_{K} 0,\|e\|_{1}=1$, and $(e, 0) \in E^{s}$. This contradiction to the first paragraph of the proof establishes that there exists a neighborhood $U^{1}$ of zero with $U^{1} \cap W^{s}(0)$ satisfying the conclusion of the theorem. The theorem now follows from properties of $W^{s}(0)$ and the fact that $\phi_{t}$ preserves $\leqq_{K}$ for $t \geqq 0$.

In Fig. 2.1 we depict a typical unstable steady state, its stable manifold and its most unstable manifold.

We end this section with some brief remarks concerning periodic orbits of type $\mathrm{K}$ monotone systems. We have already mentioned the result of Hirsch that a type $\mathrm{K}$ monotone system cannot have an attracting periodic orbit. Thus, it would appear that closed orbits are not of interest for type $\mathrm{K}$ monotone systems. They are of great interest, however, for systems for which the time reversed flow preserves a partial ordering ( $-f$ satisfies (2.3)), the so-called competitive systems which can and often do have attracting closed orbits. For this reason, this author made a study of the invariant manifold structure associated with periodic orbits of irreducible type $\mathrm{K}$ monotone systems in [52]. Briefly, in [52] we show that every closed orbit of an irreducible type $\mathrm{K}$ monotone system has a simple Floquet multiplier which exceeds unity. This largest multiplier gives rise to a "most unstable" cylinder manifold 


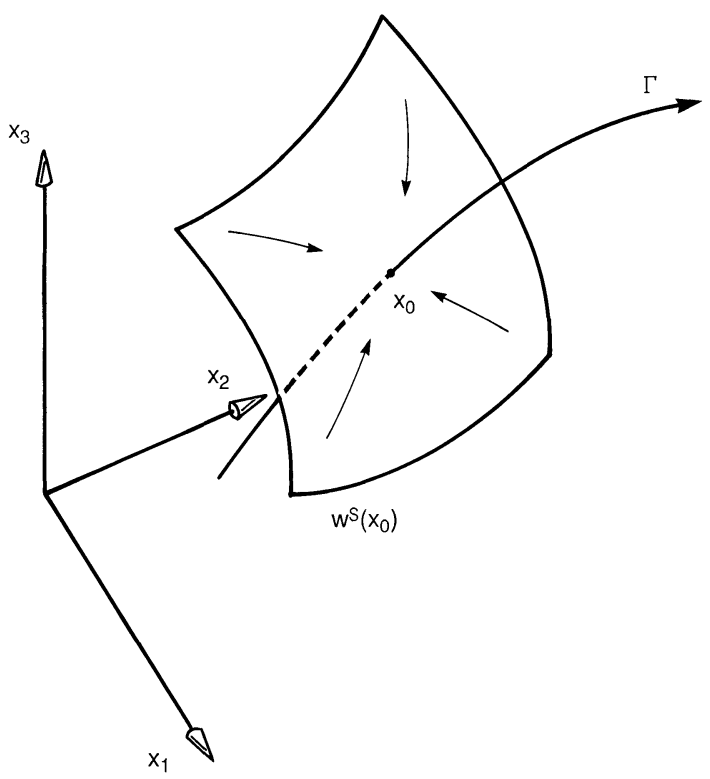

FIG. 2.1. An unstable steady state, $x_{0}$, in $R^{3}$ with $K=R_{+}^{3}, \operatorname{dim} W^{S}\left(x_{0}\right)=2$. Points of $W^{S}\left(x_{0}\right)$ are not related by and $\Gamma$ is a monotone curve.

associated with the closed orbit which has monotonicity properties. Roughly speaking, this result is the counterpart of Theorem 2.8 for steady states. It is also shown in [52] that the stable manifold of a periodic orbit (if it exists) has the property that no pair of its points can be related by $\leqq_{K}$. This, of course, is the counterpart of Theorem 2.10 for steady states.

It is an interesting application of Lemma 2.2 and the Perron-Frobenius theorem to show that a nontrivial closed orbit $\gamma$ of an irreducible type $\mathrm{K}$ monotone system must have a Floquet multiplier which is real and larger than 1. Let $p \in \gamma$ and let $T$ be the period of $\gamma$. Consider variational matrix $\partial \phi_{T} / \partial x(p)$. Clearly we have $\partial \phi_{T} / \partial x(p) f(p)=f(p)$ since $\phi_{t}^{\prime}(p)$ is a solution of the variational equation about $\phi_{t}(p)$. By Lemma $2.3(\mathrm{a})$, neither $f(p) \geqq_{K} 0$ nor $f(p) \varliminf_{K} 0$ can hold. On the other hand, by the Perron-Frobenius theorem for positive matrices, $\partial \phi_{T} / \partial x(p)$ has a positive spectral radius $\rho$ which is a simple eigenvalue and which strictly exceeds in modulus all remaining eigenvalues. Moreover, corresponding to $\rho$ there is an eigenvector $v>_{K} 0$. It follows that $\rho>1$.

3. Some special classes of type $K$ monotone systems. In this brief section we develop a simple algorithm for deciding when a particular system is type $\mathrm{K}$ monotone and which partial ordering is preserved by the corresponding flow. We also identify certain special classes of systems which are type $\mathrm{K}$ monotone.

Let us suppose that we are given an autonomous system of equations

$$
x^{\prime}=f(x)
$$

where $f$ is a smooth function defined on an open convex set $U$ in $R^{n}$. We are interested in determining if (3.1) is a type $\mathrm{K}$ monotone system. Recalling Lemma 2.1 and in particular Remark 4, we must compute $D f(x)$. First, we check the Jacobian for sign stability of the off-diagonal elements, i.e., one must establish that either $\partial f_{i} / \partial x_{j}(x) \leqq 0$ for all $x \in U$ or that $\partial f_{i} / \partial x_{j}(x) \geqq 0$ for all $x \in U$, for each $i \neq j$. Having done this, and 
assuming that (3.1) passes this test, we must next consider the requirement of sign symmetry of $D f$ in $U$. In other words, $\partial f_{i} / \partial x_{j}(x) \partial f_{j} / \partial x_{i}(y) \geqq 0$ should hold for all $i \neq j$, $x, y \in U$. This can usually be checked simply by a look at $D f$. Again, we assume that (3.1) has passed this test of sign symmetry. Now it suffices to consider only the entries of $D f$ above the main diagonal.

For each $i<j,(i, j) \in N \times N$, define $s_{i j} \in Z_{2}=(\{0,1\},+(\bmod 2))$ by the following:

$$
s_{i j}= \begin{cases}0 & \text { if } \frac{\partial f_{i}}{\partial x_{j}}>0 \text { for some } x \in U, \\ 1 & \text { if } \frac{\partial f_{i}}{\partial x_{j}}<0 \text { for some } x \in U, \\ \text { arbitrary } & \text { if } f_{i} \text { is independent of } x_{j} .\end{cases}
$$

Since we are assuming $D f$ is sign symmetric, $s_{i j}$ is well defined. Now consider the solvability of the system of $n(n-1) / 2$ equations in the $n$ unknowns $m_{i} \in Z_{2}, 1 \leqq i \leqq n$

$$
\begin{aligned}
m_{1}+m_{2} & =s_{12}, \\
m_{1}+m_{3} & =s_{13}, \\
\vdots & \\
m_{1}+m_{n} & =s_{1 n}, \\
m_{2}+m_{3} & =s_{23} \quad \text { (mod 2 arithmetic). } \\
\vdots & \\
m_{2}+m_{n} & =s_{2 n}, \\
\vdots & \\
m_{n-1}+m_{n} & =s_{n-1, n} .
\end{aligned}
$$

If there is a solution $m \in Z_{2}^{n}$ (for some choice of the $s_{i j}$ which were arbitrarily assigned), then (3.1) is type $\mathrm{K}_{m}$ monotone for this value of $m$. Equivalently, since an equation involving an arbitrarily assigned $s_{i j}$ can always be satisfied by an appropriate choice of $s_{i j}$ depending on $m_{i}$ and $m_{j}$, we can delete such equations from (3.2) and consider the solvability of the remaining equations only. Note that if (3.2) is solved by $m \in Z_{2}^{n}$ then $m+\underline{1}$ also solves (3.2) where $1=(1,1, \cdots, 1)$ since the kernel of the left-hand side of (3.2) is just $\{\underline{0}, \underline{1}\}$. This is not at all surprising since $m$ and $m+1$ correspond to orthants $K_{m}$ and $-K_{m}$ respectively and a map is easily seen to preserve one partial ordering if and only if it preserves the other.

There is a graph theoretic test for type $\mathrm{K}$ monotonicity which is described in Travis, Post and DeAngelis [38], [11], and in a private communication of Hirsch [25]. We assume $D f(x)$ is sign stable in $U$. Consider the graph $\Gamma$ with vertices 1,2 , $3, \cdots, n$. If $\partial f_{i} / \partial x_{j}$ is not identically zero in $U$, put a directed edge $E_{i j}$ from $j$ to $i$ (arrow pointing to $i$ ) and attach the sign of $\partial f_{i} / \partial x_{j}$ to the edge. Then (3.1) is type $\mathrm{K}$ monotone if and only if for every loop in $\Gamma$ the number of minus signs is even. Notice that the test for sign symmetry reduces to checking that if there is a loop consisting of an edge from $i$ to $j$ and from $j$ to $i$ then the two signs are identical. Once this has been checked, the two edges can be collapsed to a single undirected 
edge with the common sign attached. Now the signs on the edges determine the right-hand side of (3.2). It would appear then that to determine $m$ and thus the partial ordering preserved by the flow, one must do some linear algebra.

We now identify certain classes of systems whose solution operators preserve a partial ordering $\leqq_{K_{m}}$.

Example 1. Two-dimensional sign-symmetric systems. Consider a planar system (3.1) which is sign stable and sign symmetric: $\partial f_{1} / \partial x_{2} \cdot \partial f_{2} / \partial x_{1} \geqq 0$ in $U$. If both derivatives are nonnegative then (3.1) is cooperative in the usual sense. If both derivatives are nonpositive, the so-called competitive case, then (3.1) is type $\mathrm{K}_{m}$ monotone where $m=(0,1)$. In either case it is well known that all bounded solutions tend to equilibrium both as $t \rightarrow \infty$ and $t \rightarrow-\infty$ [17], [19], [24], [40].

Example 2. Three-dimensional sign-symmetric systems. Consider (3.1) where $n=3$ and sign stability holds. We assume (3.1) is sign-symmetric in $U: \partial f_{i} / \partial x_{j}$. $\partial f_{j} / \partial x_{i} \geqq 0, i \neq j$. Then (3.1) is type $\mathrm{K}_{m}$ monotone if and only if the equations

$$
\begin{aligned}
& m_{1}+m_{2}=s_{12} \\
& m_{1}+m_{3}=s_{13} \quad(\bmod 2) \\
& m_{2}+m_{3}=s_{23}
\end{aligned}
$$

are solvable in $Z_{2}^{3}$ where $\operatorname{sgn}\left(\partial f_{i} / \partial x_{j}+\partial f_{j} / \partial x_{i}\right)=(-1)^{s_{i j}}$ (the sign is to be determined at a value of $x$ in $U$ for which the quantity does not vanish if such a point exists). These equations can be solved for $m \in Z_{2}^{3}$ if and only if $s_{12}+s_{13}+s_{23}=0(\bmod 2)$. Hence (3.1) with $n=3$ and the conditions stated above is type $\mathrm{K}_{m}$ monotone if and only if

$$
\left(\frac{\partial f_{1}}{\partial x_{2}}+\frac{\partial f_{2}}{\partial x_{1}}\right)\left(\frac{\partial f_{1}}{\partial x_{3}}+\frac{\partial f_{3}}{\partial x_{1}}\right)\left(\frac{\partial f_{2}}{\partial x_{3}}+\frac{\partial f_{3}}{\partial x_{2}}\right) \geqq 0 \quad \text { in } U \text {. }
$$

The reader will recall the strong result (Theorem 2.4) stated in $\$ 2$ for threedimensional type $\mathrm{K}$ monotone systems due to Hirsch.

Example 3. Nearest neighbor interactions on a circle or line segment. Consider the following system:

$$
\begin{aligned}
x_{1}^{\prime} & =f_{1}\left(x_{n}, x_{1}, x_{2}\right), \\
x_{2}^{\prime} & =f_{2}\left(x_{1}, x_{2}, x_{3}\right), \\
& \vdots \\
x_{i}^{\prime} & =f_{i}\left(x_{i-1}, x_{i}, x_{i+1}\right), \\
& \vdots \\
& \vdots \\
x_{n}^{\prime} & =f_{n}\left(x_{n-1}, x_{n}, x_{1}\right)
\end{aligned}
$$

describing the interaction of species $x_{i}$ arranged on a circle where each species interacts only with its nearest neighbors. Note that (3.4) includes Examples 1 and 2 above as special cases.

Then $D f(x)$ is tridiagonal except for the entries $\partial f_{1} / \partial x_{n}$ in the upper right-hand corner and $\partial f_{n} / \partial x_{1}$ in the lower left-hand corner. Assuming that the $\partial f_{i} / \partial x_{j}, i \neq j$ do not change sign in $U$ and that $D f(x)$ is sign-symmetric, we will establish conditions for (3.4) to be type $K_{m}$ monotone for some $m$. Equations (3.2) reduce to 
the following:

$$
\begin{gathered}
m_{1}+m_{2}=s_{12}, \\
m_{1}+m_{n}=s_{1 n}, \\
m_{2}+m_{3}=s_{23}, \\
\vdots \\
m_{n-1}+m_{n}=s_{n-1, n} .
\end{gathered}
$$

Ignoring the second equation in (3.5) we can solve the remaining equations for $m_{2}, \cdots, m_{n}$ in terms of $m_{1}: m_{j}=s_{12}+s_{23}+s_{34}+\cdots+s_{j-1, j}+m_{1}(\bmod 2)$, $2 \leqq j \leqq n$. The second equation will be satisfied if and only if

$$
s_{1 n}+s_{12}+s_{23}+\cdots+s_{n-1, n}=0 \quad(\bmod 2) .
$$

This is the solvability condition. If it is satisfied then (3.4) is type $\mathrm{K}_{m}$ monotone for $m$ as calculated above. The solvability condition can be restated in terms of the derivatives as follows:

$$
\left(\frac{\partial f_{1}}{\partial x_{2}}+\frac{\partial f_{2}}{\partial x_{1}}\right)\left(\frac{\partial f_{2}}{\partial x_{3}}+\frac{\partial f_{3}}{\partial x_{2}}\right) \ldots\left(\frac{\partial f_{n-1}}{\partial x_{n}}+\frac{\partial f_{n}}{\partial x_{n-1}}\right)\left(\frac{\partial f_{1}}{\partial x_{n}}+\frac{\partial f_{n}}{\partial x_{1}}\right) \geqq 0 \quad \text { for all } x \in U
$$

Observe that if $\partial f_{i} / \partial x_{i+1}+\partial f_{i+1} / \partial x_{i} \equiv 0$ in $U$ for some $i \in\{0,1, \cdots, n\}$ (where $0 \approx n$ and $n+1 \approx 1$ ), then (3.6) is automatically satisfied. In this case by renumbering we may as well assume $\partial f_{1} / \partial x_{n}+\partial f_{n} / \partial x_{1} \equiv 0$ in $U$, or $f_{1}$ is independent of $x_{n}$ and $f_{n}$ is independent of $x_{1}$. We refer to such systems as sign symmetric tridiagonal systems since $D f(x)$ is a tridiagonal matrix. As we have seen, sign-symmetric tridiagonal systems are always type $\mathrm{K}_{m}$ monotone systems. Smillie [45] has obtained the following result for such systems.

THEOREM 3.1 (Smillie). Suppose $f$ is $n-1$ times differentiable and (3.1) is a signstable, sign-symmetric, irreducible, tridiagonal system. If $x(t)$ is a solution on a maximal interval $[0, a), 0<a \leqq \infty$, then either $\lim _{t \rightarrow a} x(t)$ exists and is an equilibrium point or as $t \rightarrow a, x(t)$ eventually leaves any compact set.

The irreducibility assumption for tridiagonal systems amounts to assuming that $\partial f_{i} / \partial x_{j}$ does not vanish if $|i-j|=1$. Since $-f$ satisfies the hypotheses of Theorem 3.1 if and only if $f$ does, we see that symmetric conclusions can be drawn for $x(t)$ on a maximal interval $(-b, 0], 0<b \leqq \infty$.

Finally, it may have occurred to the reader that a particular system (3.1) might have the property that its flow $\phi_{t}$ preserves several different partial orderings and perhaps the more, the better is its behavior. Certainly, if $\phi_{t}$ preserves the partial ordering generated by the orthant $K_{m}$ then it also preserves the partial ordering generated by the orthant $K_{m+1}=-K_{m}$ as we have seen. But these two partial orderings give rise to the same monotone functions and hence must be considered equivalent. The question then becomes "can a flow preserve two inequivalent partial orderings generated by orthants of $R^{n}$ ?" We show that the answer is yes but only in a trivial sort of way. Let $R^{n}=R^{k} \times R^{n-k}, x=\left(x_{1}, x_{2}\right)$ and consider the system

$$
\begin{aligned}
& x_{1}^{\prime}=f_{1}\left(x_{1}\right), \\
& x_{2}^{\prime}=f_{2}\left(x_{2}\right) .
\end{aligned}
$$


Let $\phi_{t}^{1}\left(x_{1}\right)$ and $\phi_{t}^{2}\left(x_{2}\right)$ be the solution operators of the subsystems and $\phi_{t}(x)=$ $\left(\phi_{t}^{1}\left(x_{1}\right), \phi_{t}^{2}\left(x_{2}\right)\right)$ be the solution operator for the full system. Suppose $\phi_{t}^{1}(\cdot)$ preserves a partial ordering generated by a cone $K_{m_{1}}$ in $R^{k}$ and $\phi_{t}^{2}(\cdot)$ preserves a partial ordering generated by a cone $K_{m_{2}}$ in $R^{n-k}$. Then $\phi_{t}(\cdot)$ preserves the inequivalent partial orderings generated by $K_{m_{1}} \times K_{m_{2}}=K_{\left(m_{1}, m_{2}\right)}$ and $K_{m_{1}} \times K_{-m_{2}}=K_{\left(m_{1},-m_{2}\right)}$. This rather trivial example of a system which preserves two inequivalent partial orderings is the rule. Any system (3.1) which preserves two inequivalent partial orderings generated by cones $K_{m}$ and $K_{\bar{m}}$ can be decomposed into two uncoupled subsystems. Indeed, suppose $m \neq \bar{m}$ and $m \neq-\bar{m}$ and let $T=\left\{i \in N: m_{i} \neq \bar{m}_{i}\right\}$ and $S=T^{c}$. Neither $S$ nor $T$ is empty. Since $\phi_{t}(\cdot)$ preserves the partial orderings generated by $K_{m}$ and $K_{\bar{m}}$ we have

$$
\begin{aligned}
& (-1)^{m_{i}+m_{j}} \frac{\partial f_{i}}{\partial x_{j}} \geqq 0, \quad i \neq j \\
& (-1)^{\bar{m}_{i}+\bar{m}_{j}} \frac{\partial f_{i}}{\partial x_{j}} \geqq 0, \quad i \neq j
\end{aligned}
$$

It follows immediately that

$$
\frac{\partial f_{i}}{\partial x_{j}} \equiv 0 \equiv \frac{\partial f_{j}}{\partial x_{i}} \quad \text { in } U
$$

for $(i, j) \in S \times T$. Since $U$ is convex, it follows that for $i \in S, f_{i}$ depends only on $x_{j}$ for $j \in S$ and similarly $f_{i}, i \in T$, depends only on $x_{j}$ for $j \in T$. By suitable rearrangement of equations and variables, the system must decouple as in our example.

4. Some remarks on a comparison theorem. An interesting comparison theorem has been derived by Conway and Smoller [9] and improved by Gardner [14] for systems of reaction diffusion systems which admit a bounded invariant rectangle. It turns out that this comparison technique involves type $\mathrm{K}$ monotone systems in a very natural way. We describe these results here (see [14] for a more general treatment). The reader may, if he or she chooses, consider only ordinary differential equations. Consider the system

$$
u_{t}=D \Delta u+f(u), \quad(x, t) \in \Omega \times R_{+}
$$

where $\Omega$ is a bounded domain in $R^{m}$ with smooth boundary, $u \in R^{n}$ and $f$ is a smooth map from an open set $U$ in $R^{n}$ into $R^{n}$. We do not assume $f$ has any monotonicity properties. $D$ is a constant diagonal matrix with positive diagonal elements (or $D=0$ ). We consider (4.1) together with homogeneous Neumann boundary conditions

$$
\frac{\partial u}{\partial v}=0 \quad \text { on } \partial \Omega \times R^{+}
$$

where $\partial u / \partial v=\nabla u \cdot v, v$ is the outer normal on $\partial \Omega$. It follows that solutions of the ordinary differential equation

$$
u^{\prime}=f(u)
$$

are spatially homogeneous solutions of (4.1) and (4.2). Together with (4.1) and (4.2) are prescribed continuous initial conditions

$$
u(x, 0)=u^{0}(x), \quad x \in \Omega .
$$


It will be assumed that (4.1) admits a bounded invariant rectangle

$$
\Sigma=\prod_{i=1}^{n}\left[a_{i}, b_{i}\right] \subset U
$$

in $R^{n}$ with $a_{i}<b_{i}, i \in N$. This means that if $u^{0}(x) \in \Sigma$ for $x \in \Omega$, then $u(x, t) \in \Sigma$ for all $(x, t) \in \Omega \times[0, \infty)$ for which the solution is defined. It can be shown [8] that a unique solution of (4.1), (4.2), (4.4) exists for $t \geqq 0$ and $u(x, t) \in \Sigma$ for $(x, t) \in \Omega \times[0, \infty)$ if $u^{0}(x) \in \Sigma, x \in \Omega$. Sufficient conditions for (4.1) to admit a bounded invariant rectangle are given in [8], [55] and these amount to some regularity assumptions together with the requirement that $f$ points into $\Sigma$ along the boundary of $\Sigma$ :

$$
\text { If } u \in \Sigma, \quad u_{i}=a_{i}\left(b_{i}\right) \quad \text { then } f_{i}(u) \geqq 0 \quad\left(f_{i}(u) \leqq 0\right) .
$$

Systems of the type (4.1), (4.2) are common mathematical expressions of chemical and biological phenomena, and the existence of invariant rectangles for such systems, at least for models in population biology, is also commonplace [6], [9], [14], [55]. The idea now is to construct various ordinary differential equations whose solutions bound the solutions of (4.1), (4.2) and (4.3) when $u^{0}(x) \in \Sigma$ for $x \in \Omega$.

Let $I$ be a subset of $N$ and denote by $J$ the complement of $I$ in $N$. Define the orthant $K_{I}=\left\{u \in R^{n}: u_{i} \geqq 0, i \in I, u_{j} \leqq 0, j \in J\right\}$ and write $\leqq_{I}$ for the associated partial ordering. Define $n$-vectors $A_{I}, B_{I}$ by $A_{I}^{i}=a_{i}, i \in I$ and $A_{I}^{j}=b_{j}, j \in J$ and $B_{I}^{i}=b_{i}, i \in I$ and $B_{I}^{j}=a_{j}, j \in J$. Note that $A_{I} \leqq_{I} B_{I}$ and $\Sigma=\left[A_{I}, B_{I}\right]_{I}$. Now, define functions ${ }_{I} f_{p}^{+}$, ${ }_{I} f_{p}^{-},{ }_{J} f_{p}^{+},{ }_{J} f_{p}^{-}, p \in N$, on $\Sigma$ by

$$
\begin{aligned}
& { }_{I} f_{p}^{ \pm}(u)=\left\{\begin{array}{c}
\max \\
\min
\end{array}\right\}\left\{f_{p}(\xi): \xi \in\left[A_{I}, u\right]_{I} \text { and } \xi_{p}=u_{p}\right\}, \\
& { }_{J} f_{p}^{ \pm}(u)=\left\{\begin{array}{c}
\max \\
\min
\end{array}\right\}\left\{f_{p}(\xi): \xi \in\left[u, B_{I}\right]_{I} \text { and } \xi_{p}=u_{p}\right\} .
\end{aligned}
$$

Define $H_{I}(v)=\left(h_{1}(v), \cdots, h_{n}(v)\right), v \in \Sigma$ by $h_{i}(v)={ }_{I} f_{i}^{+}(v), i \in I$, and $h_{j}(v)={ }_{I} f_{j}^{-}(v)$, $j \in J$. Define $H_{J}(v)=\left(h_{1}(v), \cdots, h_{n}(v)\right)$ by $h_{i}(v)={ }_{J} f_{i}^{-}(v), i \in I$ and $h_{j}(v)={ }_{J} f_{j}^{+}(v)$, $j \in J$. The vector function $H_{I}$ and $H_{J}$ are Lipschitz continuous on $\Sigma$ (see [9], [55]) and it is not difficult to see that

$$
H_{J}(u) \leqq_{I} f(u) \leqq_{I} H_{I}(u), \quad u \in \Sigma .
$$

Let $v_{I}(t)$ be a solution of

$$
\begin{array}{ll}
v^{\prime}=H_{I}(v), & v(0)=v_{I}^{0} \in \Sigma, \\
u^{0}(x) \leqq_{I} v_{I}^{0}, & x \in \Omega,
\end{array}
$$

and for the complementary set $J$, let $v_{J}(t)$ be a solution of

$$
\begin{array}{ll}
v^{\prime}=H_{J}(v), & v(0)=v_{J}^{0} \in \Sigma, \\
v_{J}^{0} \leqq_{I} u^{0}(x), & x \in \Omega .
\end{array}
$$

With these definitions, we may state the result due to Gardner [14] in the following way.

THEOREM 4.1. For all $(x, t) \in \Omega \times R_{+}$we have

$$
v_{J}(t) \leqq_{I} u(x, t) \leqq_{I} v_{I}(t) .
$$

It is easily seen that $\Sigma$ is positively invariant for the systems (4.9) and (4.10). It may appear that we are merely introducing some different notation for a known 
result. The following result may justify this notational change. Let $\phi_{t}^{I}$ and $\phi_{t}^{J}$ be the solution maps for (4.9) and (4.10).

THEOREM 4.2. The systems (4.9) and (4.10) are type $K_{I}$ monotone. Moreover, we have for $t \geqq 0$

$$
A_{I} \leqq_{I} \phi_{t}^{J}\left(A_{I}\right) \leqq_{I} v_{J}(t) \leqq_{I} u(x, t) \varliminf_{I} v_{I}(t) \varliminf_{I} \phi_{t}^{I}\left(B_{I}\right) \varliminf_{I} B_{I} .
$$

The map $t \rightarrow \phi_{t}^{J}\left(A_{I}\right)$ is monotone nondecreasing with respect to $\leqq_{I}$ and $\phi_{t}^{J}\left(A_{I}\right)$ tends to an equilibrium of (4.10) as $t \rightarrow \infty$. Similarly, the map $t \rightarrow \phi_{t}^{I}\left(B_{I}\right)$ is nonincreasing and $\phi_{t}^{I}\left(B_{I}\right)$ tends to an equilibrium of (4.9) as $t \rightarrow \infty$.

Before proceeding to the proof of Theorem 4.2, a point of clarification should be made. Since $H_{I}$ is only Lipschitz continuous in general, (4.9) cannot be type $\mathrm{K}$ monotone in the sense of $\$ 2$. However, as pointed out in Remark 3 of $\S 2$, the flow $\phi_{t}^{I}$ of (4.9) can preserve the partial ordering $\geqq_{I}$ for $t \geqq 0$ under weaker hypotheses. The appropriate hypothesis on a vector function $h$ is the following [7], [51].

$$
\begin{aligned}
& \text { If } v, w \in \Sigma \text { with } v \leqq_{I} w \text { and } v_{l}=w_{l} \text { for some } l \\
& \text { then } h_{l}(v) \leqq h_{l}(w) \text { if } l \in I \text { and } h_{l}(v) \geqq h_{l}(w) \text { if } l \in J .
\end{aligned}
$$

Proof of Theorem 4.2. We must check that $(\mathrm{H})$ holds for $H_{I}$ and $H_{J}$. We consider $H_{J}$ only. For $i \in I, H_{J}^{i}(v)={ }_{J} f_{i}^{-}(v)$. Clearly, if $v \leqq_{I} w, v_{i}=w_{i},{ }_{J} f_{i}^{-}(v) \leqq_{J} f_{i}^{-}(w)$ since the minimum is being taken over a smaller set in defining ${ }_{J} f_{i}^{-}(w)$. For $j \in J$, $H_{J}^{j}(v)={ }_{J} f_{j}^{+}(v)$ so $f_{j}^{+}(v) \geqq_{J} f_{j}^{+}(w)$ if $v \leqq_{I} w, v_{j}=w_{j}$ since the maximum is being taken over a smaller set in defining ${ }_{J} f_{j}^{+}(w)$. Hence $(\mathrm{H})$ holds for $H_{J}$ and $H_{J}$ is type $\mathrm{K}_{I}$ monotone by [51, Thm. 2.4].

The inequality $A_{I} \leqq_{I} \phi_{t}^{J}\left(A_{I}\right)$ follows from the positive invariance of $\Sigma$ for (4.10). The inequality $\phi_{t}^{J}\left(A_{I}\right) \leqq_{I} v_{J}(t)$ follows from the fact that (4.10) is type $\mathrm{K}_{I}$ monotone and that $A_{I} \leqq_{I} v_{J}(0)$. The other inequalities come from Theorem 4.1 or can be established in a similar manner.

The monotonicity of $\phi_{t}^{J}\left(A_{I}\right)$ follows immediately from the fact that $A_{I} \leqq_{I} \phi_{t}^{J}\left(A_{I}\right)$ for $t \geqq 0$ and since (4.1) is type $\mathrm{K}_{I}$ monotone. This completes our proof.

Theorem 4.2 is essentially giving us an idea of what one can expect to accomplish through the use of the comparison technique. Since we know that the typical solution of a type $\mathrm{K}$ monotone system converges to an equilibrium as $t$ tends to infinity, we can expect that $v_{J}(t)$ and $v_{I}(t)$ will converge to equilibria of (4.10) and (4.9), respectively. If we label these equilibria by $Q$ and $P, v_{J}(t) \rightarrow Q$ and $v_{I}(t) \rightarrow P$, then the comparison technique yields that the omega limit set of the orbit through $u^{0}$ consists of functions taking values in $[Q, P]_{I} \subset \Sigma$. We might hope that $P=Q$ in which case $u(x, t)$ converges to an equilibrium of (4.3) uniformly for $x \in \Omega(f(Q)=0$ by (4.8)).

Let us denote by $\bar{A}_{I}=\lim _{t \rightarrow \infty} \phi_{t}^{J}\left(A_{I}\right)$ and $\bar{B}_{I}=\lim _{t \rightarrow \infty} \phi_{t}^{I}\left(B_{I}\right)$ the equilibria of (4.10) and (4.9), respectively, described in Theorem 4.2. If $A_{I}<_{I} \bar{A}_{I} \leqq_{I} \bar{B}_{I}<_{I} B_{I}$, then we may set $\Sigma^{\prime}=\left[\bar{A}_{I}, \bar{B}_{I}\right]_{I}$ and attempt to iterate the comparison technique by redefining $H_{I}$ and $H_{J}$ relative to $\Sigma^{\prime}$. This is essentially the technique used by Brown [6], who has nicely refined the comparison technique in order to obtain convergence of $u(x, t)$ to a constant equilibrium of (4.1), (4.2). Brown [6] assumes (4.1), (4.2) has a oneparameter family of "contracting rectangles" $\Sigma(\tau)=[a(\tau), b(\tau)], 0 \leqq \tau \leqq 1$, i.e., rectangles on the boundary of which the inequalities (4.6) are strict, with $\Sigma(0)=\Sigma$, $\Sigma(1)=\left\{u^{*}\right\}$, an equilibrium. Furthermore, it is assumed that $a(\tau)$ is increasing and $b(\tau)$ is decreasing, both continuous functions of $\tau$. Under these assumptions, Brown shows that if $u^{0}(x) \in \Sigma(0)$ then $u(x, t) \rightarrow u^{*}$ as $t \rightarrow \infty$ uniformly in $x \in \Omega$.

The comparison technique described above should be particularly effective when the system (4.3) is a type $\mathrm{K}_{m}$ monotone system. In this case, set $I=\left\{i \in N: m_{i}=0\right\}$ and $J=\left\{j \in N: m_{j}=1\right\}$. Then it is easy to see that $f(v)=H_{I}(v)=H_{J}(v)$ (see 4.8). 
Corollary 4.3. If (4.3) is type $K_{m}$ monotone and $I, J$ as above, then $f(v)=H_{I}(v)=H_{J}(v), v \in \Sigma$. If $\Sigma$ belongs to the domain of attraction of a steady state $u^{*}$ of (4.3), then $u(x, t) \rightarrow u^{*}$ as $t \rightarrow \infty$ uniformly in $x \in \Omega$ whenever $u^{0}(x) \in \Sigma, x \in \Omega$.

Corollary 4.3 has the following interesting implication. If $g$ is a type $\mathrm{K}_{I}$ monotone vector field defined on $\Sigma$ such that $f(u) \leqq_{I} g(u)$ for $u \in \Sigma$, then $H_{I}(u) \leqq_{I} g(u)$ for $u \in \Sigma$. Hence $H_{I}(u)$ is the minimal type $\mathrm{K}_{I}$ monotone vector field satisfying the right-hand inequality of (4.8). Similarly, $H_{J}(u)$ is the maximal type $\mathrm{K}_{I}$ monotone vector field satisfying the left-hand inequality of (4.8).

Corollary 4.3 explains the very successful application of the comparison technique to the case where (4.3) is a planar competitive or symbiotic system given by Conway and Smoller [9]. In the following section we will apply Corollary 4.3 to a mathematical model of cyclic gene systems involved in the control of protein synthesis. We mention that the comparison technique is applicable to the single loop feedback systems studied by Selgrade [40] and to mathematical models of mutualism [50] and mutualism and competition [51].

Finally, it should be mentioned that many other authors have contributed to the understanding of monotonicity in reaction diffusion systems. Notable among these are Martin [32] and Matano [33].

5. An application. In this section we apply some of the results of the previous sections to a mathematical model of a repressible cyclic gene system following Banks and Mahaffy [3], [4]. We will consider here only a very special case of the model which is treated in considerably more detail by the author in [54]. It is assumed that two genes control the synthesis of certain proteins. The first gene is transcribed producing mRNA, $\left(y_{1}\right)$, which in turn is translated to produce a protein, $\left(y_{2}\right)$, which acts as an enzyme for the production of protein $\left(y_{3}\right)$ and so on until an end product protein $y_{p}$ is produced. This last protein acts as a repressor, inhibiting the transcription of the second gene. A similar sequence of reactions, leading from the second gene, leads to the production of proteins $z_{1}, \cdots, z_{l}$, the last of which acts as a repressor inhibiting the transcription of the first gene (see Fig. 5.1).

The letters $y_{i}$ and $z_{j}$ will also be used to denote the concentrations of the proteins and thus are nonnegative quantities. An appropriate system of differential equations is given by

$$
\begin{aligned}
& y_{1}^{\prime}=f_{1}\left(z_{l}\right)-\alpha_{1} y_{1}, \\
& y_{i}^{\prime}=y_{i-1}-\alpha_{i} y_{i}, \\
& z_{1}^{\prime}=f_{2}\left(y_{p}\right)-\beta_{1} z_{1}, \\
& z_{j}^{\prime}=z_{j-1}-\beta_{j} z_{j}, \quad 2 \leqq i \leqq p,
\end{aligned}
$$

The parameters of $\alpha_{i}, \beta_{j}$ are positive and the functions $f_{i}$ are positive-valued, strictly decreasing smooth functions vanishing at infinity. After suitable scaling, we assume

$$
f_{i}(v)>0, \quad f_{i}^{\prime}(v)<0, \quad f_{i}(0)=1, \quad f_{i}(\infty)=0, \quad i=1,2 .
$$

We can apply the graphical test for type K monotonicity of (5.1). Note that there is exactly one loop in the graph associated with (5.1) (see \$3) and this loop contains two negative feedbacks corresponding to the two nonlinearities $f_{1}$ and $f_{2}$. It follows that (5.1) is type $\mathrm{K}$ monotone.

The state of the system is given by the vector $u=(y, z) \in R_{+}^{p+l}$. We leave to the reader the verification that $(5.1)$ is type $\mathrm{K}$ monotone where $K$ is the orthant $K=R_{+}^{p} \times\left(-R_{+}^{l}\right)$. 


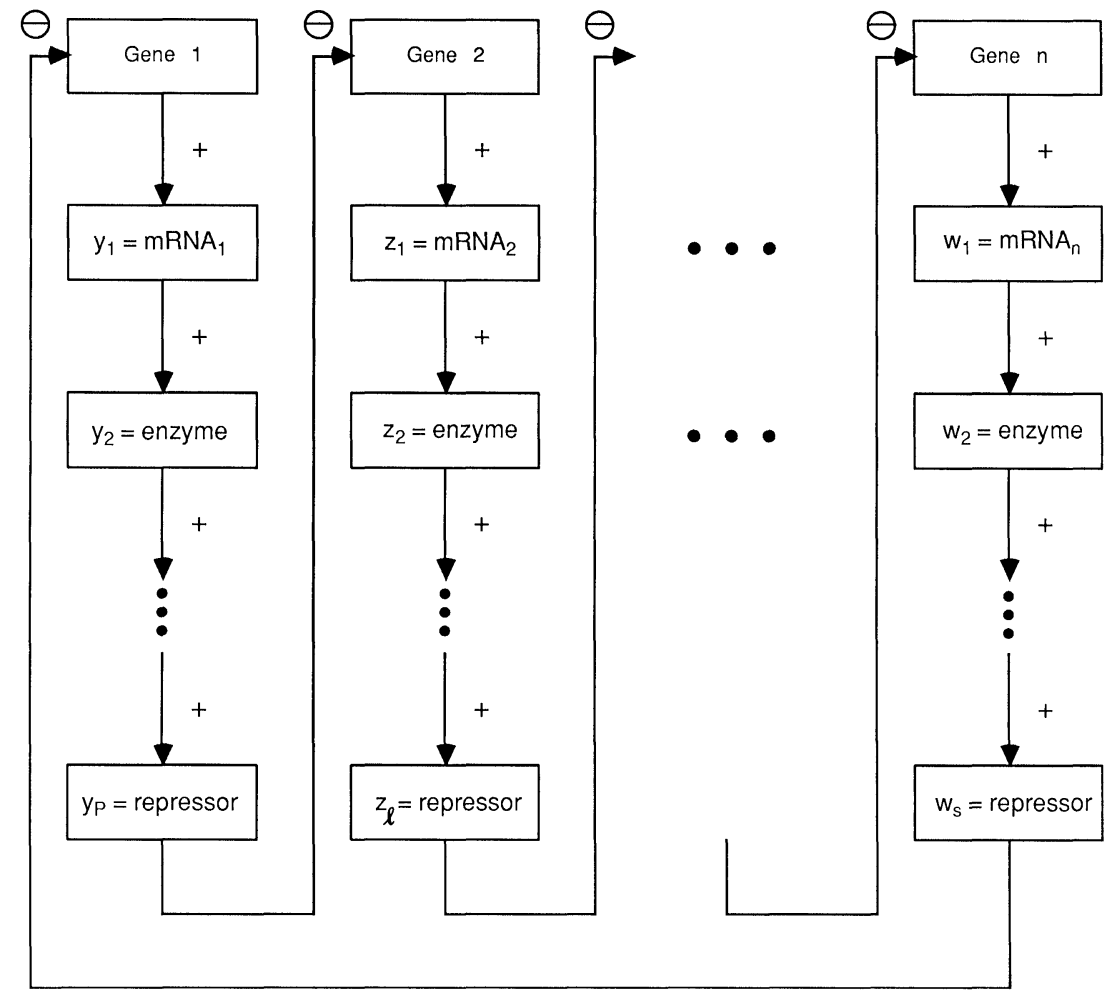

FIG. 5.1. A repressible cyclic gene system.

If we denote by $F$ the vector field defined by (5.1), then we have

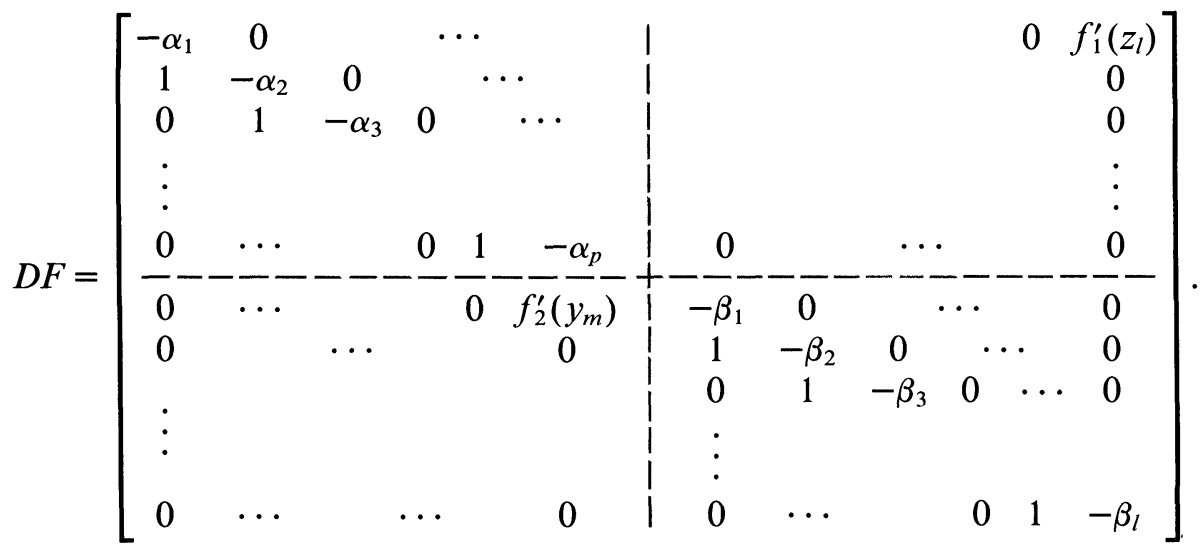

The loop of nonvanishing terms $a_{1 m}, a_{m, m-1}, a_{m-1, m-2} \cdots a_{21}, a_{1 m}$ in the matrix $D F=\left(a_{i j}\right)_{i, j=1}^{m}, m=p+l$, implies that $D F$ is irreducible and hence (5.1) is an irreducible type $\mathrm{K}$ monotone system.

Equilibria of (5.1) are given in terms of $y_{p}$ and $z_{l}$ by

$$
\begin{aligned}
& y_{p-1}=\alpha_{p} y_{p}, \quad y_{p-2}=\alpha_{p} \alpha_{p-1} y_{p}, \cdots, \quad y_{1}=\alpha_{p} \alpha_{p-1} \cdots \alpha_{2} y_{p}, \\
& f_{1}\left(z_{l}\right)=\gamma_{1} y_{p}, \quad \gamma_{1} \equiv \alpha_{1} \alpha_{2} \alpha_{3} \cdots \alpha_{p}, \\
& z_{l-1}=\beta_{l} z_{l}, \quad z_{l-2}=\beta_{l} \beta_{l-1} z_{l}, \cdots, \quad z_{1}=\beta_{l} \beta_{l-1} \cdots \beta_{2} z_{l}, \\
& f_{2}\left(y_{p}\right)=\gamma_{2} z_{l}, \quad \gamma_{2} \equiv \beta_{1} \beta_{2} \cdots \beta_{l} .
\end{aligned}
$$


Hence steady states of (5.1) are in one-to-one correspondence with nonnegative solutions of

$$
\begin{aligned}
& \gamma_{1}^{-1} f_{1}\left(z_{l}\right)=y_{p}, \\
& \gamma_{2}^{-1} f_{2}\left(y_{p}\right)=z_{l},
\end{aligned}
$$

or, equivalently, of solutions of

$$
g\left(z_{l}\right) \equiv\left(\gamma_{2}^{-1} f_{2} \circ \gamma_{1}^{-1} f_{1}\right)\left(z_{l}\right)=z_{l} .
$$

In view of our assumptions on $f_{i}, g:[0, \infty) \rightarrow[0, \infty)$ satisfies $g(0)=\gamma_{2}^{-1} f_{2}\left(\gamma_{1}^{-1}\right)$, $g^{\prime}\left(z_{l}\right)>0$ for $z_{l}>0, g(\infty)=\gamma_{2}^{-1}$.

For simplicity, we assume without further mention that

(5.5) If $g(z)=z$ then $g^{\prime}(z) \neq 1$.

It then follows that $g$ has an odd finite number, $r$, of fixed points $z^{1}<z^{2} \cdots<z^{r}$ and (5.1) has $r$ steady states:

$$
\begin{aligned}
& \bar{u}_{s}=\left(\bar{y}^{s}, \bar{z}^{s}\right), \quad s=1,2, \cdots, r, \\
& \bar{y}_{i}^{s}=\left(\alpha_{1} \cdots \alpha_{i}\right)^{-1} f_{1}\left(z^{s}\right), \quad i=1, \cdots, p, \\
& \bar{z}_{j}^{s}=\gamma_{2}\left(\beta_{1} \beta_{2} \cdots \beta_{j}\right)^{-1} z^{s}, \quad j=1,2, \cdots, l .
\end{aligned}
$$

Since $f_{1}$ is strictly decreasing it follows that the set of steady states is a totally ordered set (see Fig. 5.2):

$$
\bar{u}_{r}<_{K} \bar{u}_{r-1}<_{K} \cdots \bar{u}_{2}<_{K} \bar{u}_{1} .
$$

The stability of a steady state $\bar{u}$ can be inferred from Theorem 2.7. Simply take absolute value of the off-diagonal elements of (5.3) and check that the principal



Fig. 5.2. The steady states of (5.1) in case $r=5$. The shaded regions belong to the domains of attraction of the odd indexed equilibria. 
minors of the resulting matrix alternate in sign. It is easily seen that principal minors alternate in sign except possibly for the largest minor, the determinant. Thus $s(D F(\bar{u}))<0$ if and only if

$$
\gamma_{1} \gamma_{2}-f_{1}^{\prime}\left(z_{l}\right) f_{2}^{\prime}\left(y_{m}\right)>0 \text { or equivalently } g^{\prime}\left(z_{l}\right)<1 .
$$

From the characteristic polynomial equation

$$
\prod_{i=1}^{p}\left(\lambda+\alpha_{i}\right) \prod_{j=1}^{l}\left(\lambda+\beta_{j}\right)-f_{1}^{\prime}\left(z_{l}\right) f_{2}^{\prime}\left(y_{m}\right)=0
$$

we see that (5.6) is just the condition that the constant term in the polynomial is positive. Also note that (5.5) and the form of the characteristic polynomial imply that $s(D F(\bar{u})) \neq 0$; in fact $D F(\bar{u})$ is nonsingular, so either $\bar{u}$ is asymptotically stable or there is at least one eigenvalue of $D F(\bar{u})$ with positive real part and $\bar{u}$ is unstable.

It is interesting that the existence and stability of steady states of (5.1) is mirrored by the existence and stability of fixed points of the map $g$. In particular, the odd indexed $\bar{u}_{i}$ are asymptotically stable and the even indexed $\bar{u}_{i}$ are unstable.

LEMmA 5.1. For each $\eta \geqq 1, \xi \geqq 1$ the box

$$
D(\eta, \xi)=\prod_{i=1}^{p}\left[0, \eta\left(\alpha_{1} \cdots \alpha_{i}\right)^{-1}\right] \times \prod_{j=1}^{l}\left[0, \xi\left(\beta_{1} \cdots \beta_{j}\right)^{-1}\right]
$$

is positively invariant. Moreover for each fixed $\eta>1$ and $\xi>1$, every solution of $(5.1)$ eventually enters and remains in $D(\eta, \xi)$.

Proof. The positive invariance of $D(\eta, \xi)$ follows immediately from the fact that $F$ points into $D(\eta, \xi)$ along the boundary of $D(\eta, \xi)$ (use $0 \leqq f_{i} \leqq 1$ ). Now fix $\eta>1$ and $\xi>1$. Since $y_{1}^{\prime} \leqq 1-\alpha_{1} y_{1}$ it follows that $y_{1}(t)<\alpha_{1}^{-1} \eta$ for large $t$. Hence for large $t, y_{2}^{\prime}<\alpha_{1}^{-1} \eta-\alpha_{2} y_{2}$ and so $y_{2}(t)<\left(\alpha_{1} \alpha_{2}\right)^{-1} \eta$ for sufficiently large $t$. As we continue in this fashion, the last assertion becomes obvious.

We let $D \equiv D(1,1)$. The following result can be inferred from the results of $\S 2$ (see [54]).

THEOREM 5.2. If $B\left(\bar{u}^{i}\right)$ denotes the basin of attraction of $\bar{u}^{i}$ in $R_{+}^{p+l}$, then

$$
\cup_{i \text { odd }} B\left(\bar{u}^{i}\right)
$$

is open and dense in $R_{+}^{p+l}$. If $r=1$ then $\bar{u}_{1}$ is globally attracting in $R_{+}^{p+l}$.

Theorem 5.2 follows essentially from Lemma 5.1 and Theorem 2.6. One can obtain more information concerning the basins of attraction by very simple arguments. Namely the following inclusions hold:

$$
\begin{aligned}
& \left\{u \in R_{+}^{p+l}: u \leqq_{K} \bar{u}_{r-1}, u \neq \bar{u}_{r-1}\right\} \subset B\left(\bar{u}_{r}\right) \\
& \left\{u \in R_{+}^{p+l}: \bar{u}_{r-1} \leqq_{K} u \leqq_{K} \bar{u}_{r-3}, u \neq \bar{u}_{r-1}, \bar{u}_{r-3}\right\} \subset B\left(\bar{u}_{r-2}\right) \\
& \quad \vdots \\
& \left\{u \in R_{+}^{p+l}: \bar{u}_{4} \leqq u \leqq \bar{u}_{2}, u \neq \bar{u}_{4}, \bar{u}_{2}\right\} \subset B\left(u_{3}\right) \\
& \left\{u \in R_{+}^{p+l}: \bar{u}_{2} \leqq_{K} u, u \neq \bar{u}_{2}\right\} \subset B\left(\bar{u}_{1}\right) .
\end{aligned}
$$

We establish the last inclusion only; the others follow similarly. Since $s\left(D F\left(\bar{u}_{2}\right)\right)>0$, Theorem 2.8 implies the existence of a monotone trajectory emanating 
from $\bar{u}_{2}$ and tending to $\bar{u}_{1}$ as $t$ tends to infinity. In addition, Theorem 2.8 implies that $\left\{u: \bar{u}_{2} \leqq_{K} u \leqq_{K} \bar{u}_{1}, u \neq u_{2}\right\}$ belongs to $B\left(\bar{u}_{1}\right)$. Note that if $u, v \in B\left(\bar{u}_{1}\right), u \leqq_{K} v$, then $[u, v]_{K} \subset B\left(\bar{u}_{1}\right)$ by the order preserving property of the flow. Our remarks following the statement of Theorem 2.5 imply that if $v \geqq_{K} 0$, then the set of points $u$ on the half line $\left\{u: u=u_{1}+t v, t \geqq 0\right\}$ such that the positive orbit through $u$ does not tend to a steady state is at most a countable set. Now if $u$ lies on this half line then $u \geqq_{K} \bar{u}_{1}$ so $\phi_{t}(u) \geqq_{K} \bar{u}_{1}$ for all $t>0$. Thus $\bar{u}_{1}$ is the only steady state that $\phi_{t}(u)$ can tend to as $t$ tends to infinity. It now is apparent from the previous two remarks that for every point, $u$, on the half line, $\phi_{t}(u)$ tends to $\bar{u}_{1}$ as $t$ tends to infinity. Since $v \geqq_{K} 0$ is arbitrary, we have established that $\left\{u: u \geqq_{K} \bar{u}_{1}\right\} \subset B\left(\bar{u}_{1}\right)$. The last inclusion follows by sandwiching a point $u$ with $u \geqq_{K} \bar{u}_{2}, u \neq u_{2}$, between a point in $\left\{u: \bar{u}_{2} \leqq_{K} u \leqq_{K} \bar{u}_{1}, u \neq \bar{u}_{2}\right\}$ and a point in $\left\{u: \geqq_{K} \bar{u}_{1}\right\}$ and applying a previous remark.

\section{REFERENCES}

[1] G. Aronsson AND R. B. KellogG, On a differential equation arising from compartmental analysis, Math. Biosci., 38 (1978), pp. 113-122.

[2] G. Aronsson And I. Mellander, A deterministic model in biomathematics: Asymptotic behavior and threshold conditions, Math. Biosci., 49 (1980), pp. 207-222.

[3] H. T. BANKS AND J. M. MAHAFFY, Mathematical models for protein biosynthesis, LCDS TR 79-4, Div. Appl. Math., Brown University, Providence, RI, 1979.

[4] — Stability of cyclic gene models for systems involving repression, J. Theoret. Biol., 74 (1978), pp. 323-324.

[5] A. Berman AND R. J. Plemmons, Nonnegative Matrices in the Mathematical Sciences, Academic Press, New York, 1979.

[6] P. N. BRown, Decay to uniform states in ecological interactions, SIAM J. Appl. Math., 38 (1980), pp. 22-37.

[7] L. P. Burton AND W. M. Whyburn, Minimax solutions of ordinary differential systems, Proc. Amer. Math. Soc., 3 (1952), pp. 794-803.

[8] K. N. Cheuch, C. C. Conley AND J. A. Smoller, Positively invariant regions for systems of nonlinear diffusion equations, Indiana Univ. Math. J., 26 (1977), pp. 373-392.

[9] E. D. Conway AND J. A. Smoller, A comparison technique for systems of reaction-diffusion equations, Comm. Partial Differential Equations, 7 (1977), pp. 679-697.

[10] W. A. Coppel, Stability and Asymptotic Behavior of Differential Equations, Heath Mathematical Monographs, New York, 1965.

[11] D. L. DeAngelis, W. M. Post and C. C. Travis, Positive Feedback in Natural Systems, SpringerVerlag, Berlin, Heidelberg, New York, 1986.

[12] M. FIEDLER AND B. PTAK, On matrices with non-positive off-diagonal elements and positive principal minors, Czechoslovak Math. J., 12 (1962), pp. 382-400.

[13] F. R. GantmaCher, The Theory of Matrices, vol. 2, 1959, Chelsea, New York.

[14] R. GARDNER, Comparison and stability theorems for reaction diffusion systems, SIAM J. Math. Anal., 12 (1980), pp. 603-616.

[15] B. S. GoH, Sector stability of a complex ecosystem model, Math. Biosci., 40 (1978), pp. 157-166.

[16] —, Stability in models of mutualism, Amer. Natur., 113 (1979), pp. 261-275.

[17] S. Grossberg, Competition, decision and concensus, J. Math. Anal. Appl., 66 (1978), pp. 470-493.

[18] J. K. Hale AND A. S. Somolinos, Competition for fluctuating nutrient, J. Math. Biol., 18 (1983), pp. 255-280.

[19] M. W. Hirsch, Systems of differential equations which are competitive or cooperative I: Limit sets, SIAM J. Math. Anal., 13 (1982), pp. 167-179.

[20] — Systems of differential equations which are competitive or cooperative II: Convergence almost everywhere, SIAM J. Math. Anal., 16 (1985), pp. 432-439.

[21] - The dynamical systems approach to differential equations, Bull. Amer. Math. Soc., 11 (1984), pp. 1-64.

[22] —_, Differential equations and convergence almost everywhere in strongly monotone flows, Contemporary Math., 17, Amer. Math. Soc., Providence, RI, pp. 267-285. 
[23] - Stability and convergence in strongly monotone dynamical systems, J. Reine Angew. Math., to appear.

[24] M. W. Hirsch and S. Smale, Differential Equations, Dynamical Systems, and Linear Algebra, Academic Press, New York, 1974.

[25] M. W. HiRSCH, Private communication.

[26] C. D. Johnson, Sufficient conditions for D-stability, J. Econom. Theory, 9 (1974), pp. 53-62.

[27] E. Kamke, Zur Theorie der Systeme gewöhnlicher differential Gleichungen, II, Acta Math., 58 (1932), pp. 57-85.

[28] M. A. KRASNOSEL'SKII, Translation along Trajectories of Differential Equations, Vol. 19, Trans. Math. Monographs, Amer. Math. Soc., Providence, RI, 1968.

[29] H. KUIPER, Existence and comparison theorems for nonlinear diffusion systems, J. Math. Anal. Appl., 60 (1977), pp. 166-181.

[30] A. LAJMANOVICH AND J. YORKE, A deterministic model for gonorrhea in a nonhomogeneous population, Math. Biosci., 28 (1976), pp. 221-236.

[31] V. Lakshmikantham and S. Leela, Differential and Integral Inequalities, Vol. 1, Academic Press, New York, 1969.

[32] R. H. Martin, JR., Asymptotic stability and critical points for nonlinear quasimonotone parabolic systems, J. Differential Equations, 30 (1978), pp. 391-423.

[33] H. MATANo, Existence of nontrivial unstable sets for equilibriums of strongly order preserving systems, J. Fac. Sci. Univ. Tokyo, 30 (1984), pp. 645-673.

[34] P. De Mottoni AND A. SChiaffino, Competition systems with periodic coefficients: a geometric approach, J. Math. Biol., 11 (1981), pp. 319-355.

[35] M. MüLLER, Über das Fundamentaltheorem in der Theorie der gewöhnlichen Differentialgleichungen, Math. Z., 26 (1926), pp. 619-645.

[36] H. ОтнмеR, The qualitative dynamics of a class of biochemical control circuits, J. Math. Biol., 3 (1976), pp. 53-78.

[37] G. Poole AND T. Boullion, A survey on M-Matrices, this Review, 16 (1974), pp. 419-427.

[38] W. M. Post, C. C. Travis and D. L. DeAngelis, Mutualism, limited competition and positive feedback, Biology of Mutualism, Ecology and Evolution, D. Boucher, ed., to appear.

[39] D. SATTINGER, Monotone methods in nonlinear elliptic and parabolic equations, Indiana Univ. Math. J., 21 (1972), pp. 979-1000.

[40] J. SeLgRADE, Asymptotic behavior of solutions to single loop positive feedback systems, J. Differential Equations, 38 (1980), pp. 80-103.

[41] — A Hopf bifurcation in single-loop positive-feedback systems, Quart. Appl. Math. (1982), pp. 347-351.

[42] - On the existence and uniqueness of connecting orbits, Nonlinear Anal., 7 (1983), pp. 11231125.

[43] D. D. SiljaK, Large-Scale Dynamic Systems, Stability and Structure, North-Holland, New York, 1978.

[44] S. Smale, On the differential equations of species in competition, J. Math. Biol., 3 (1976), pp. 5-7.

[45] J. SMILlie, Competitive and cooperative tridiagonal systems of differential equations, SIAM J. Math. Anal., 15 (1984), pp. 530-534.

[46] H. L. SMITH, Periodic solutions of periodic competitive and cooperative systems, SIAM J. Math. Anal., 17 (1986), pp. 1289-1318.

[47] - Periodic competitive differential equations and the discrete dynamics of competitive maps, J. Differential Equations, 64 (1986), pp. 165-194.

[48] - Cooperative systems of differential equations with concave nonlinearities, Nonlinear Anal., 10 (1986), pp. 1037-1052.

[49] - Invariant curves for mappings, SIAM J. Math. Anal., 17 (1986), pp. 1053-1067.

[50] - On the asymptotic behavior of a class of deterministic models of cooperating species, SIAM J. Appl. Math., 46 (1986), pp. 368-375.

[51] - Competing subcommunities of mutualists and a generalized Kamke theorem, SIAM J. Appl. Math., 46 (1986), pp. 856-874.

[52] — Periodic orbits of competitive and cooperative systems, J. Differential Equations, 65 (1986), pp. 361-373.

[53] — Monotone semiflows, generated by functional differential equations, J. Differential Equations, 66 (1987), pp. 420-442.

[54] — Oscillation and multiple steady states in a cyclic gene model with repression, J. Math. Biol., 25 (1987), pp. 169-190.

[55] J. A. SMOLLER, Shock Waves and Reaction-Diffusion Equations, Springer-Verlag, New York, 1982. 
[56] Y. TAKeUChI AND N. ADACHI, The existence of globally stable equilibria of ecosystems of the generalized Volterra type, J. Math. Biol., 10 (1980), pp. 401-415.

[57] Y. TAKeuchi, N. Adachi and H. Tokumaru, Global stability of ecosystems of the generalized Volterra type, Math. Biosci., 42 (1978), pp. 119-136.

[58] C. C. Travis and W. M. Post III, Dynamics and comparative statics of mutualistic communities, J. Theoret. Biol., 78 (1979), pp. 553-571.

[59] W. Walter, Differential and Integral Inequalities, Springer-Verlag, Berlin, Heidelberg, New York, 1970. 